\title{
Cocoa Extract Exerts Sex-Specific Effects in an Aggressive Hy- per-Glycemia Model: A Pilot Study
}

\author{
Kathryn C. Racine ${ }^{1}$, Lisard Iglesias-Carres ${ }^{1}$, Jacob A. Herring ${ }^{2,3}$, Mario G. Ferruzzi ${ }^{1,4}$, Colin D. Kay ${ }^{1}$, Jeffery S. Tes- \\ sem $^{2}$, Andrew P. Neilson ${ }^{*}$
}

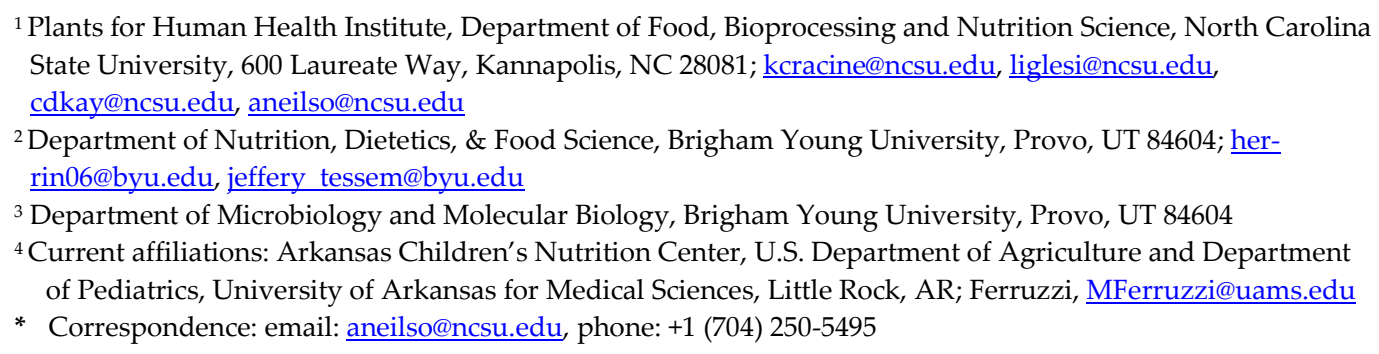

\begin{abstract}
Type 2 diabetes (T2D) is characterized by hyperglycemia and insulin resistance. Cocoa may slow T2D development and progression. This study employed male and female BTBR.Cg-

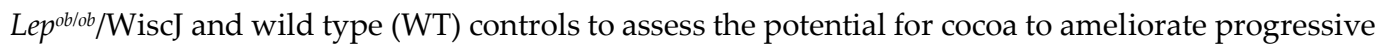
$\mathrm{T} 2 \mathrm{D}$ and compare responses between sexes. Mice received diet without (WT, ob/ob) or with cocoa extract $(o b / o b+c)$ for 10 weeks. Glucose and insulin tolerance tests (GTT/ITT) were conducted at weeks 1, 5 and 2, 6, respectively. Cocoa provided mild non-significant protection against weight gain vs. $o b / o b$ control in males but not females. Male $o b / o b+c$ had increasing fasting glucose at weeks 1 and 5 GTTs, with significantly higher levels of fasting glucose than $o b / o b$ control at week 5 . This was not seen in females. Cocoa protected against elevated 4-hour fasting glucose in week 2, but not week 6, ITTs. Cocoa partly suppressed hyperinsulinemia in males but significantly amplified it in females and protected against loss of beta cell area in females only. The mechanisms of these sexspecific effects remain to be elucidated. This study informs additional experiments with larger sample sizes and demonstrates that sex differences must be considered when designing dietary interventions for T2D.
\end{abstract}

Keywords: BTBR, ob/ob, type-2 diabetes, flavanol, insulin, beta cell

\section{Introduction}

Diabetes is a health crisis, imposing great public health and socio-economic burdens globally. Type 1 diabetes (T1D) is preceded by inflammation and an autoimmune response leading to pancreatic beta cell destruction, whereas type 2 diabetes (T2D) is often preceded by inflammation and chronic metabolic stress as well as pancreatic beta cell dysfunction [1]. While beta cell dysfunction is traditionally viewed as a late-stage event in T2D, growing data suggest that beta cell dysfunction may be an early event [2-4]. The International Diabetes Federation reported in 2019 that approximately 463 million adults worldwide were living with diabetes [5]. It is estimated that $90 \%$ of cases are T2D, with another 374 million people at risk of developing T2D by 2045 [5]. Mismanagement of T2D results in chronic complications, including cardiovascular disease, kidney failure, neuropathy, and retinopathy. It is critical to develop effective strategies for T2D prevention and management. Obesity is one of the most prominent risk factors for T2D, as it can result in progressive insulin resistance [6,7]. In an insulin resistant state, perceived insulin demand is heightened, resulting in increased stress on pancreatic beta cells- ultimately increasing beta cell mass and disrupting the balance of proliferation and apoptosis [8]. This combination of hyperinsulinemia and dysregulated beta cell physiology is, in large part, the precursor to beta cell exhaustion and failure and ultimately hypoinsulinemia in late-stage T2D.

Flavanols have drawn interest for their potential role in T2D prevention and management [9-11]. Cocoa (Theobroma cacao) is one of the most concentrated dietary sources of flavanols [12-14]. Numerous preclinical studies have 
suggested a potential anti-diabetic role for cocoa flavanols [15]. The majority of these studies focus on obesity, hyperglycemia, and insulin resistance. Few studies have examined the impact of cocoa flavanols on beta cell function. Pancreatic beta cells secrete insulin and are responsible for maintenance of glucose homeostasis, along with glucagonsecreting alpha cells. Physiological conditions accompanying T2D, such as chronic oxidative stress and inflammation, eventually induce beta cell failure and death and accelerate the progression of disease [6]. Promising studies suggest that cocoa and other sources of flavanols protect beta cells in vitro [8,16-19] and in vivo [20-22], but this has not been investigated as thoroughly as protective effects in adipose tissue and skeletal muscle. We previously demonstrated that bioavailable monomeric flavanols enhance beta cell function by increasing mitochondrial respiration [23]. Human studies of cocoa in the context of obesity and hyperglycemia have had mixed results [24-28]. To effectively harness the potential benefits of cocoa in humans, more information from preclinical models is needed regarding the specific contexts (disease state, genetics, and sex) in which cocoa may be effective.

Rodent T2D models include insulin resistance and beta cell failure phenotypes. Popular monogenic models include Lep ${ }^{o b / o b}$ ("ob/ob"), Lepr ${ }^{d b / d b}$ (" $\left.d b / d b^{\prime \prime}\right)$, and Zucker diabetic fatty rats (ZDF). These models are deficient in leptin signaling and exist in a state of perceived starvation, consuming excess food but suppressing energy expenditure [29]. The $d b / d b$ and ZDF models develop hyperglycemia and diabetes around 8-10 weeks of age whereas $o b / o b$ are hyperglycemic by 4 weeks [30]. Environmental models include high fat feeding and/or streptozotocin (STZ) administration. High fat feeding mirrors human disease more accurately than genetic models but involves slow development of beta cell damage, while STZ rapidly induces hyperglycemia by damaging pancreatic beta cells. Low-dose STZ along with high fat diet is an accelerated T2D model [30]. In this pilot study, we aimed to employ an aggressive T2D model with the potential to reach beta cell exhaustion and failure. To achieve this, the ob/ob mutation on the novel BTBR (black and tan, brachyuric) background (BTBR.Cg-Lep ob/WiscJ) was used with wild type (WT) controls. Although data on T2D progression in BTBR $o b / o b$ mice are limited, a few groups have compared this model with $o b / o b$ mice on the C57BL/6J (B6) background genome [29,31-34]. In BTBR background ob/ob mice, there are significant genetic differences in tissues that play a key role in glucose metabolism response in obesity, compared to B6 background mice. BTBR $o b / o b$ mice progress more rapidly to late-stage T2D and experience a loss of specific microRNAs (miRNAs) in pancreatic islet cells, potentially resulting in an inability to increase beta cell replication in response to obesity that happens on the B6 background [35]. BTBR ob/ob mice have significantly higher food intake and gain significantly more weight than B6 ob/ob, and display glycosuria [29].

Our objectives were to perform a pilot study to 1) determine the potential for cocoa to ameliorate progressive $\mathrm{T} 2 \mathrm{D}$, and 2) compare sex differences in the response of severely T2D mice to cocoa supplementation. We hypothesized that cocoa supplementation would protect against progressive T2D, but that efficacy would differ based on sex.

\section{Results}

2.1 Animal genotyping. Results from genotyping of fixed liver tissue are presented in Supplementary Table 1. Animals were ordered and shipped directly from Jax and through this analysis it was identified that one animal (male, $o b / o b+c$ (C)) did not carry the $o b$ mutation on either allele. This mouse has been removed from analysis and the male $o b / o b+c$ treatment thus has $n=2$.

2.2 Cocoa. Composition measures of cocoa extract are shown in Table 1. The yield of CE from powder was $18 \%$, which was higher than the anticipated $10 \%$ that was used to calculate diet formulations. The estimated total polyphenol and total flavanol content of the extract were $0.25 \pm 0.008 \mathrm{mg} \mathrm{GAE} / \mathrm{mg}$ CE and $0.200 \pm 0.013 \mathrm{mg}$ PCB2 equivalents/mg CE, respectively. The mDP of all flavanols was $2.14 \pm 0.024 \mathrm{mDP}$. The $\mathrm{mDP}$ of flavanols other than monomeric flavanols was $2.55 \pm 0.048$. UPLC-MS/MS analysis of individual procyanidins is described in Table 1 . The values reported in these analyses generally agree with our previous analyses of cocoa extract [36,37]. 
Table 1: Cocoa extract characterization

\begin{tabular}{lcc}
\hline \multicolumn{2}{c}{ Measure } & \multicolumn{1}{c}{ Value } \\
\hline $\begin{array}{l}\text { Total polyphenols } \\
\left(\mathrm{mg} \mathrm{GAE} / \mathrm{mg} \text { extract } \pm \text { SEM }{ }^{\mathrm{a}}\right)\end{array}$ & (Folin) & $0.25 \pm 0.01$ \\
\hline $\begin{array}{l}\text { Total flavanols } \\
\left(\mathrm{mg} \text { PCB2/mg extract } \pm \mathrm{SEM}^{\mathrm{b}}\right)\end{array}$ & (DMAC) & $0.20 \pm 0.01$ \\
\hline $\begin{array}{l}\text { Mean degree of polymerization (thiolysis) } \\
(\mathrm{mDP} \pm \mathrm{SEM})^{\mathrm{c}}\end{array}$ & $\begin{array}{c}\text { Including monomers: } 2.14 \pm 0.02 \\
\text { Excluding monomers: } 2.55 \pm 0.05\end{array}$ \\
\hline
\end{tabular}

\begin{tabular}{lc}
\hline Procyanidin characterization & (LC-MS/MS) \\
(mg/g extract \pm SEM) & $8.56 \pm 0.10$ \\
Catechin & $18.9 \pm 0.36$ \\
Epicatechin & $5.69 \pm 0.16$ \\
Dimer & $6.74 \pm 0.23$ \\
Trimer & $3.93 \pm 0.043$ \\
Tetramer & $3.61 \pm 0.078$ \\
Pentamer & $1.61 \pm 0.11$ \\
Hexamer & $1.27 \pm 0.14$ \\
Heptamer & $0.664 \pm 0.027$ \\
Octamer & $0.376 \pm 0.041$ \\
Nonamer & $0.119 \pm 0.019$ \\
Decamer & \\
\hline aGallic acid equivalents & \\
bProcyanidin B2 equivalents & \\
cMean degree of polymerization (monomer residues per flavan-3-ol molecule)
\end{tabular}

2.3 Body weights and food intake. Weight gain (as a \% of initial weight) and food intake are presented in Figure 1 (male: A-C, and female: D-F). Absolute weights are presented in Supplementary Figure 1. WT male and female mice on the CE-free control diet experienced very similar weight gain, approximately $12 \mathrm{~g}$ total ( 30\% increase of initial body weight) over $10 \mathrm{wks}$ (Figures 1B, E). Interestingly, the homozygotes $(o b / o b)$ fed the control diet exhibited significant variation in weight gain in both sexes, with weight gains of $\sim 2-30 \mathrm{~g}(\sim 0-50 \%$ increase of initial weight). It is unclear whether this phenotypic variation is typical on the BTBR background, but it is possible that this variation could be partially due to weight loss (or, in this case, lack of weight gain despite $o b / o b$-induced hyperphagia) that may be exhibited in severely diabetic animals and humans [38-40]. WT male and female had a larger \% weight gain, when expressed as \% of initial (Figures $1 \mathrm{~B}$ and E), compared to $o b / o b$ controls but this is likely a factor of the higher initial weights of $o b / o b$ in both sexes (Supplementary Figure $1 \mathrm{~A}, \mathrm{C}$ ). While no statistically significant differences were observed (due to small sample size and large variability in the $o b / o b$ on the control diet), CE supplementation appeared to have distinct effects in male vs. female mice: CE appeared to slightly reduce weight gain in males, with no such trend (or slight gain) apparent in females. The $o b / o b$ mice for both sexes ate more food compared to WT controls (Figures $1 \mathrm{C}$ and F), consistent with the known hyperphagia phenotype in $o b / o b$ mice. Importantly, the inclusion of CE did not appear to reduce food intake in $o b / o b$ mice, indicating that observed changes in body composition, metabolism, or other markers in the CE treatment were not due to reduced caloric intake. This finding has been reported before for equivalent doses of intact cocoa [41,42]. Based on the average food intake and body weights over the course of the study for each sex (male: 1082 and $1224 \mathrm{~g}$ diet/kg body weight/week in $o b / o b$ vs. $o b / o b+c$, respectively; female: 1114 and $1270 \mathrm{~g}$ diet/kg body weight/day in $o b / o b$ vs. $o b / o b+c$, respectively), the average intake of CE was approximately $9791 \mathrm{mg} \mathrm{CE} / \mathrm{kg}$ body weight/week for male $o b / o b+\mathrm{c}$ and $10163 \mathrm{mg} \mathrm{CE} / \mathrm{kg}$ body weight/week for female $o b / o b+\mathrm{c}$. Given our actual CE yield of 18\% from cocoa powder, these CE intakes correspond to doses of flavanols equivalent to 54300-56500 mg whole cocoa powder/kg cage weight/week. Although we did not measure intake daily, assuming 
equal distribution over time, this corresponds 7800-8100 mg cocoa powder $/ \mathrm{kg}$ body weight $/ \mathrm{d} \sim 1400-1450 \mathrm{mg} \mathrm{CE} / \mathrm{kg}$ body weight/d (Supplementary Figure 2). This dose corresponds to previous work, reporting approximately $5700 \mathrm{mg}$ cocoa powder/kg body weight/d, when utilizing C57BL/6J mice- a model that consumes lower quantities of food than that of the $o b / o b$ strain utilized in this pilot study [42].

\section{Male}
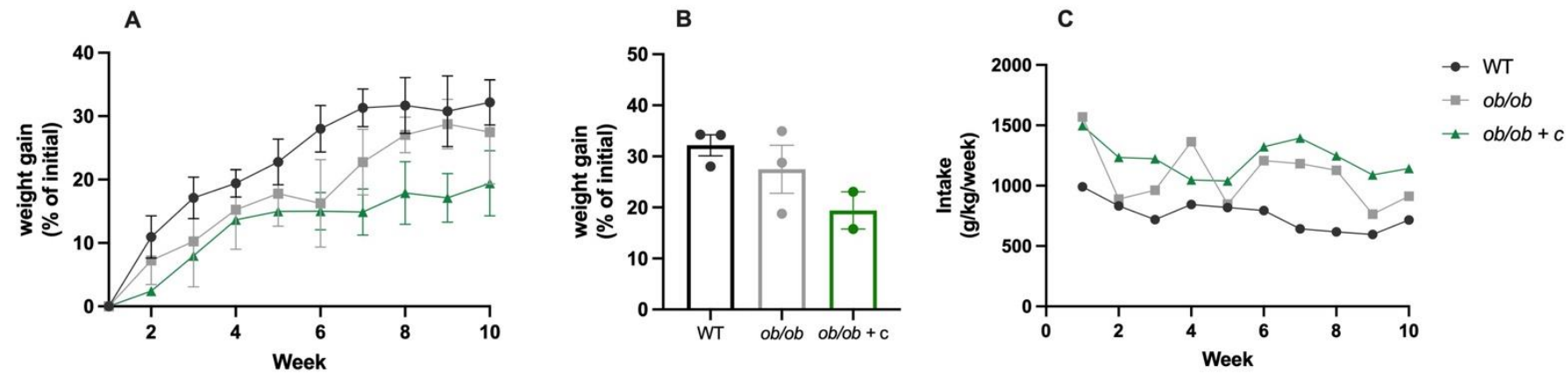

\section{Female}

D

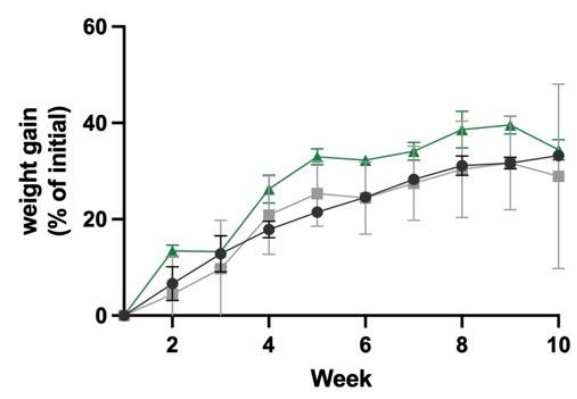

E

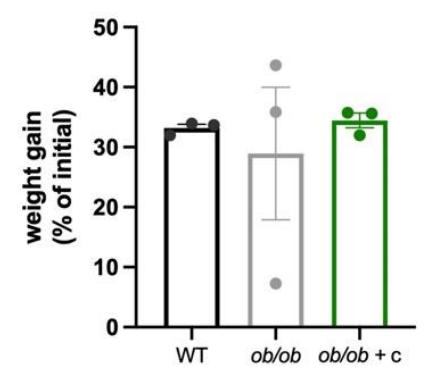

$\mathbf{F}$

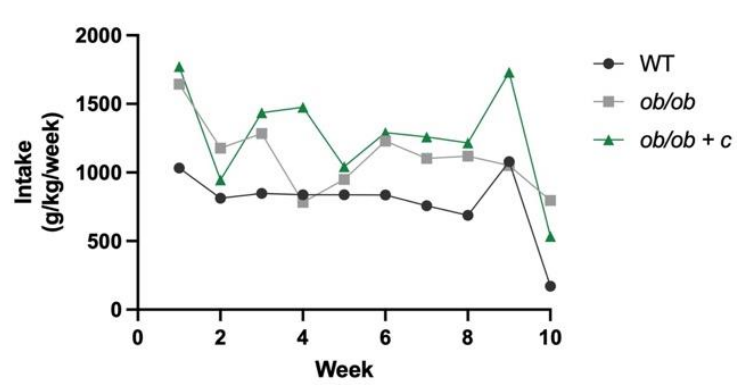

Figure 1. Weight gain and food intake during the 10-week experiment for male (A-C) and female (D-F) mice. Male mice: weight gain over time (\% of initial) $(A)$, total weight gain as a \% of initial (B) and food intake over time (C). Female mice: weight gain over time (\% of initial) (D), total weight gain as a \% of initial $(E)$ and food intake over time $(F)$. Dots represent individual animals; colored bars and error bars represent mean \pm SEM. For bar graphs, data were analyzed by 1-way ANOVA; due to lack of observed overall treatment effect for any graph, no post hoc tests were performed to compare treatment means. Error bars and statistical analyses are not shown for food intake, as each value is from $n=1$ cage.

2.4 Glycemic control. GTT results from weeks 1 and 5 are shown in Figure 2. Fasting blood glucose values are shown in Figure 2A and B. Although not statistically significant due to the small sample size, the fasting blood glucose values of the $o b / o b$ control mice had already begun to separate themselves from the WT controls at week 1 of the study (week 5 of age overall). Fasting glucose values for $o b / o b+c$ were not significantly different from $o b / o b$ controls but were significantly elevated compared to WT controls at week 1 in males, but not females. It is important to note that for week 1 measurements, only 1.5-2 days had passed since the start of dietary intervention. While fasting blood glucose values did not change for WT mice between weeks 1 and 5 for either sex, values for $o b / o b$ controls and $o b / o b+c$ groups significantly worsened in both sexes over the same period. At week 5 , the male fasting blood glucose values were significantly different for all groups, with levels for $o b / o b+c$ greater than those for the $o b / o b$ controls, which in turn were greater than WT controls. The trends in males indicate that fasting blood glucose worsened in $o b / o b$ mice over time, as expected. Data for female mice were more intriguing. As expected, fasting blood glucose for both ob/ob treatments worsened over time. While not statistically significant, there was an indication towards reduced fasting blood glucose in the $o b / o b+c$ group compared to the $o b / o b$ controls at both 1 and 5 weeks in females. 

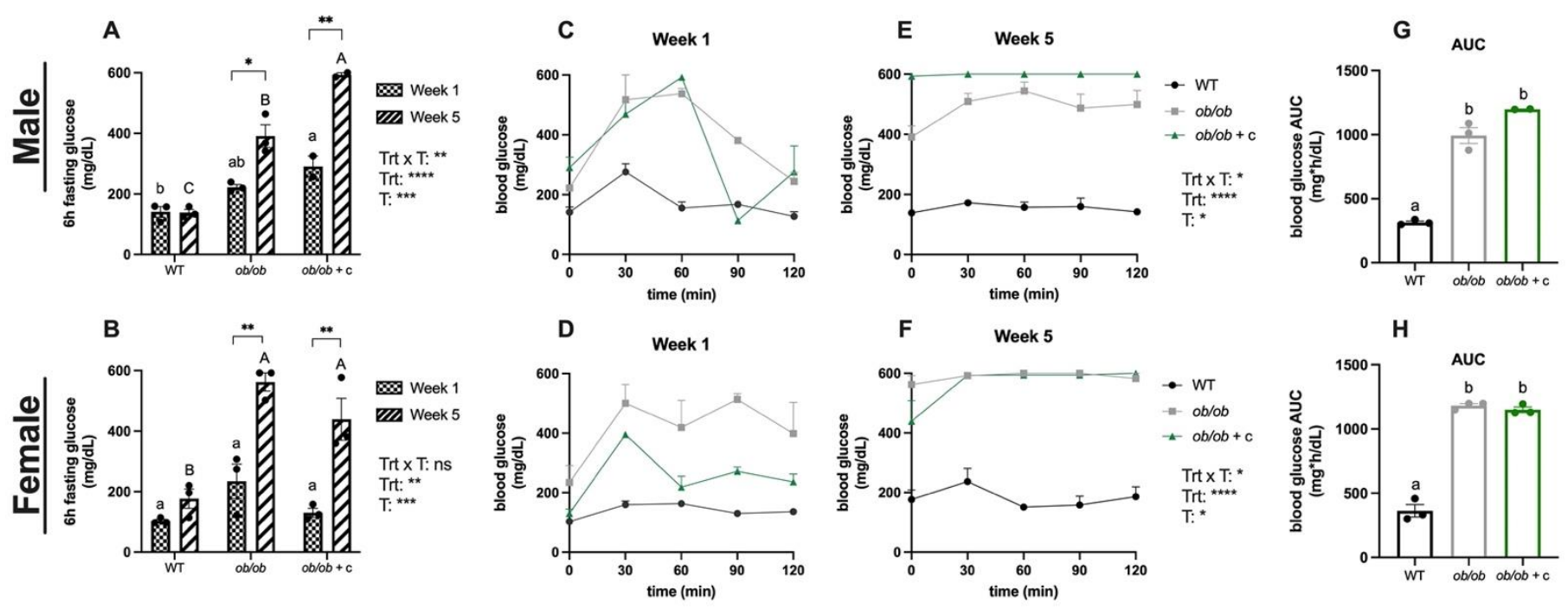

Figure 2. Glucose tolerance test measures. Six hour fasting blood glucose levels prior to glucose tolerance tests at weeks 1 and 5 for male $(A)$ and female $(B)$ mice. Blood glucose excursion curves during i.p. glucose tolerance tests at weeks 1 and 5 for male (C,E) and female $(D, F)$ mice. Dots represent individual animals; colored bars and error bars represent mean \pm SEM. Data were analyzed by 2-way ANOVA: treatment (Trt), time ( $T$, time 0-120 min or week 1 vs. 5), and significance of main effects/interactions are shown in inset boxes for each graph. If a significant main effect or interaction was detected, Sidak (time) or Tukey's (treatment) post hoc tests for multiple comparisons were performed: ${ }^{*}, * * * *$ and $* * *$ indicate $P \leq 0.05, P \leq 0.01, P \leq 0.001$ and $P \leq 0.0001$, respectively. Lower-case and upper-case superscript letters indicate significance for week 1 and week 5 , respectively. Graphs without inset boxes did not show significant main effects/interactions. Blood glucose area under the curve (AUC) for week 5 GTT (G, H). Values are presented as mean \pm SEM. Data were analyzed by 1-way ANOVA. If a significant treatment effect was detected, Tukey's post hoc test was performed to compare treatment means. Bars not sharing a common superscript letter are significantly different $(P<0.05)$. It must be noted that the maximum glucometer reading was $600 \mathrm{mg} / \mathrm{dL}$ and therefore in some cases the true glucose reading could not be determined. The apparent lack of variability among high readings is not necessarily representative of a lack of biological variability, but rather a limitation in the instrumentation utilized.

Blood glucose excursion curves for GTTs at weeks 1 and 5 are shown in Figures 2C-F. Male and female WT had minimal induction of hyperglycemia across week 1 and week 5 GTT measurements compared to $o b / o b$ and $o b / o b+c$ treatments. At week 1, although only supplemented with cocoa for 2 days, cocoa supplementation appeared to significantly improve blood glucose clearance (at $90 \mathrm{~min}$ ) in males and generally reduce blood glucose in females at all time points compared to $o b / o b$ controls, suggesting an acute effect. Male $o b / o b$ had lower blood glucose levels at $2 \mathrm{~h}$ compared to female $o b / o b$ at week 1 . At week 5, glycemic control had significantly worsened for both male $o b / o b$ and $o b / o b$ + c compared to week 1. CE supplementation appeared to worsen glycemic control at week 5 for males, as blood glucose levels were higher for $o b / o b+c$ compared to $o b / o b$; AUCs were not significantly different for these 2 groups, although $o b / o b+c$ appeared slightly higher. Similarly, glycemic control had significantly worsened for both female $o b / o b$ and $o b / o b+c$ compared to week 1. Interestingly, glycemic control appeared worse for $o b / o b$ females compared to males at week 5 . Note that the apparent lack of variability and flat glucose curves for the female $o b / o b$ and male $o b / o b+c$ mice at week 5 is due to the large number of readings above the measurable scale of the glucometer $(600 \mathrm{mg} / \mathrm{dL} \mathrm{maximum})$, which were all recorded as $600 \mathrm{mg} / \mathrm{dL}$ for calculation purposes. It is unknown how high these values were, and actual differences, if present, were not detectable due to the limitations of the glucometer. Week 5 GTT maximum blood glucose level $\left(\mathrm{C}_{\max }\right)$ and excursion from baseline values are illustrated in Supplementary Figure 3.

2.5 Insulin sensitivity. ITT results from weeks 2 and 6 are shown in Figure 3. Fasting blood glucose values are shown in Figures 3A, B. For males, glucose was elevated in $o b / o b$ and $o b / o b+c$ mice compared to WT controls at 2 and 6 weeks. In females, distinct effects on fasting blood glucose were observed compared to males. At 2 weeks, ob/ob females had significantly elevated fasting glucose compared to WT controls, but supplementation with CE completely prevented this increase (this trend was observed, but did not reach significance, at week 1 GTT in females, Figure 1B). At week 6, 

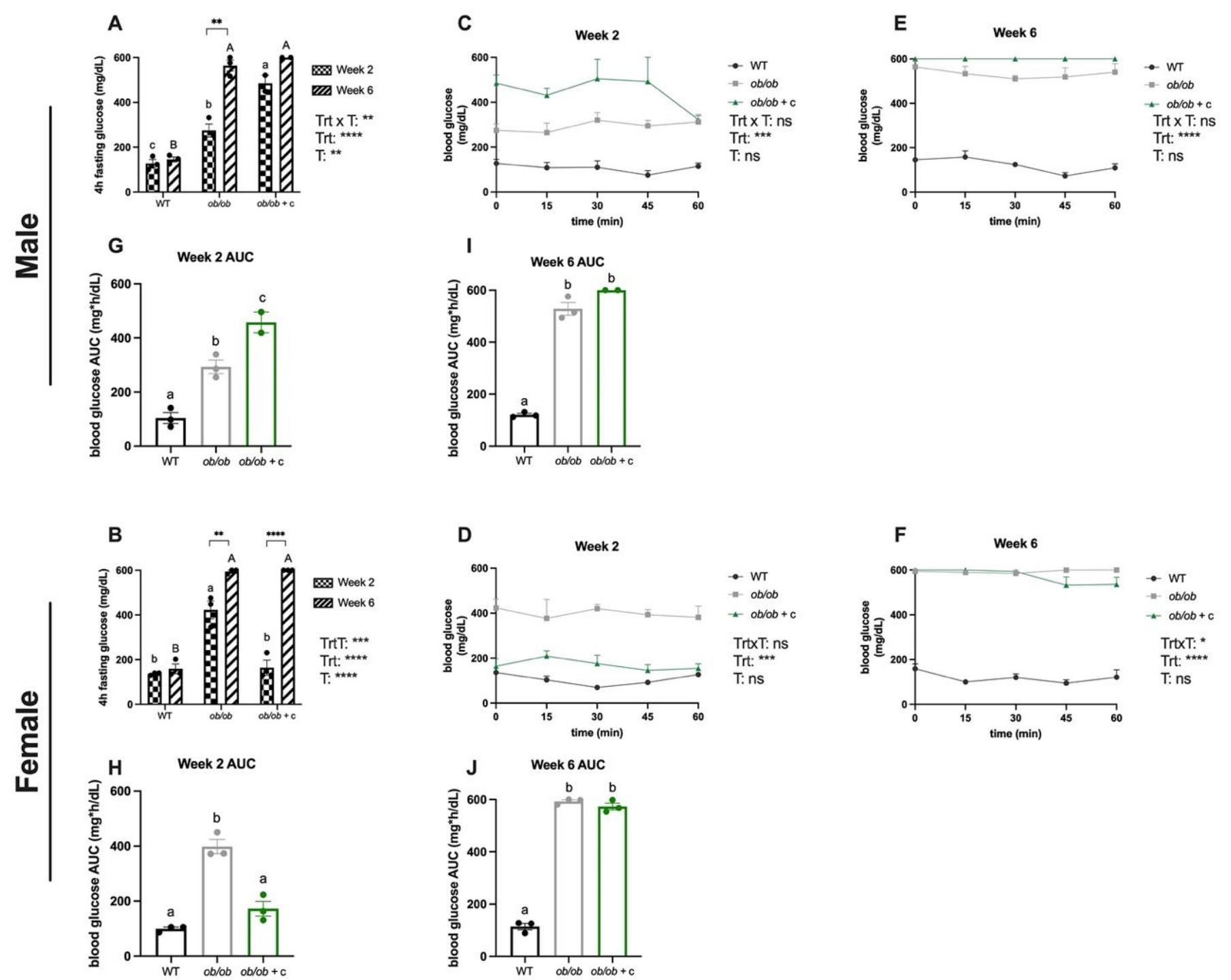

Figure 3. Insulin tolerance test measures. Four hour fasting blood glucose levels prior to insulin tolerance tests at week 2 and 6 for male $(A)$ and female $(B)$ mice. Blood glucose curves during i.p. insulin tolerance tests at weeks 2 and 6 for male $(C, E)$ and female $(D, F)$ mice. Dots represent individual animals; colored bars and error bars represent mean \pm SEM. Data were analyzed by 2-way ANOVA: treatment (Trt), time (T, 0-60 min or week $1 \mathrm{vs.}$ ), and significance of main effects/interactions are shown in inset boxes for each graph. If a significant main effect or interaction was detected, Sidak (time) or Tukey's (treatment) post hoc test for multiple comparisons were performed: ${ }^{*}, * *, * * *$ and ${ }^{* * * *}$ indicate $P \leq 0.05, P \leq 0.01, P \leq 0.001$ and $P \leq 0.0001$, respectively. Lower-case and upper-case superscript letters indicate significance for week 1 and week 5, respectively. Blood glucose area under the curve (AUC) for week $2(G, H)$ and 6 ITT $(I, J)$. Values are presented as mean $\pm S E M$. Data were analyzed by 1-way ANOVA. If a significant treatment effect was detected, Tukey's post hoc test was performed to compare treatment means. Bars not sharing a common superscript letter are significantly different $(P<0.05)$. It must be noted that the maximum glucometer reading was $600 \mathrm{mg} / \mathrm{dL}$ and therefore in some cases the true glucose reading could not be determined. The apparent lack of variability among high readings is not necessarily representative of a lack of biological variability, but rather a limitation in the instrumentation utilized.

fasting blood glucose had significantly worsened for both $o b / o b$ and $o b / o b+c$, and the protective effect of CE supplementation observed at 2 weeks was lost. It is worth noting that some differences between Figures 2 and 3 are likely due to the $6 \mathrm{~h}$ fast for GTTs (Figure 2) vs. the $4 \mathrm{~h}$ fast for ITTs (Figure 3). Again, the apparent lack of variability for the female $o b / o b$ and $o b / o b+c$ mice at week 6 is due to the large number of readings above the measurable scale of the glucometer as described above.

Blood glucose curves for ITTs at weeks 2 and 6 are shown in Figures 3C-F. For males, both ob/ob groups were less insulin sensitive than WT controls at week 2, and the $o b / o b+c$ group appeared less insulin sensitive than ob/ob controls. At week 6, both $o b / o b$ groups had worsened, and again $o b / o b+c$ group appeared less inulin sensitive than 
$o b / o b$ controls. For females, both $o b / o b$ groups were less insulin sensitive than WT controls at week 2, but CE supplementation appeared to exert significant protection against insulin resistance compared to $o b / o b$ controls. At week 6, both the $o b / o b$ and $o b / o b+c$ group had worsened, but the $o b / o b+c$ group did appear more sensitive than the $o b / o b$ group at later times in the challenge (45-60 min). Taken together, the impact of CE supplementation on insulin sensitivity was completely distinct between males and females.

Glucose AUCs for week 2 and 6 ITTs are shown in Figures 3G-J. For males at week 2, ob/ob control mice had significantly worse AUCs compared to WT mice, and CE supplementation worsened AUC. AUCs worsened for both groups of $o b / o b$ mice by week 6, and CE supplementation appeared to slightly but non-significantly worsen AUC. For females, CE supplementation blunted the elevation in AUC seen in $o b / o b$ controls at week 2, but AUCs worsened in both groups by weeks 6 and the protective effects of CE were no longer observed (protective effects may have been present, but as the glucometer could not read $>600 \mathrm{mg} / \mathrm{dL}$, such protection was not detectable).

2.6 Blood insulin levels. Fasting blood insulin levels at euthanasia are shown in Figure 4. In males, fasting insulin levels were significantly elevated in $o b / o b$ mice compared to WT controls, and this effect was completely reversed by CE supplementation. The opposite effect was observed in females: fasting insulin levels were significantly elevated in $o b / o b$ mice compared to WT controls, and CE supplementation further increased these levels by $>2$-fold. A striking sex-specific response to cocoa appears evident for fasting blood insulin levels. It should be noted that these extreme insulin values were unexpected, and for some $o b / o b+c$ females the absorbance values were $\sim 2.5 \mathrm{x}$ higher than the maximal standard curve concentrations (insufficient sample remained to re-run the assay on diluted samples). Therefore, these values are extrapolated and should be treated with caution. Nevertheless, these values suggest sex-specific responses and the extremely high insulin values suggest that, despite prolonged severe hyperglycemia, these animals were still hyperinsulinemic and had not yet progressed to appreciable beta cell failure in terms of insulin secretion capacity.

Male

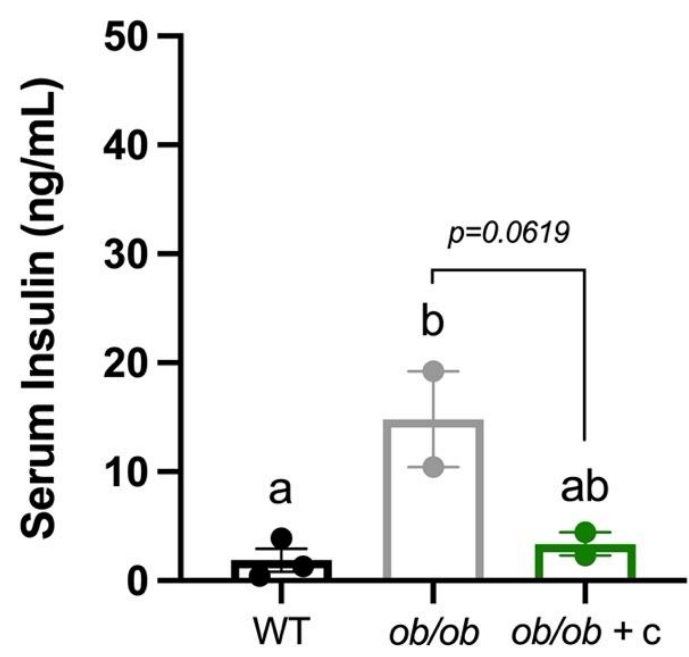

Female

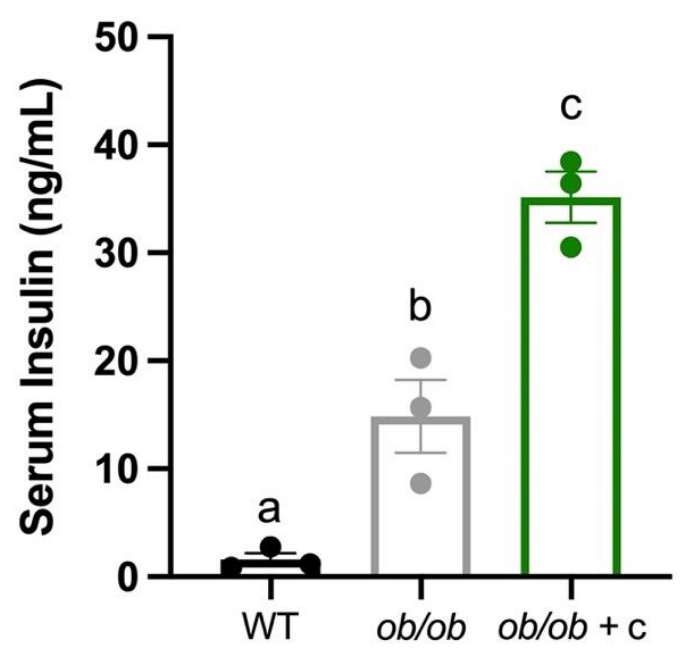

Figure 4. Fasting blood insulin levels following 10 weeks of treatment for male (A) and female (B) mice. Values are presented as mean \pm SEM. Data were analyzed by 1-way ANOVA. If a significant treatment effect was detected, Tukey's post hoc test was performed to compare treatment means. Bars not sharing a common superscript letter are significantly different $(P<0.05)$.

\subsection{Gut permeability.}

Sex-specific effects were also observed for gut permeability markers. Serum levels of zonulin, a circulating regulator of gut permeability, and colonic protein levels of the tight junction protein zona occludens-1 (ZO-1) are presented in Supplementary Figure 4. Male $o b / o b$ mice had almost complete loss of zonulin compared to WT controls, which was partially (albeit non-significantly) blunted by CE administration. Female $o b / o b$ and WT controls had similar zonulin levels, and CE supplementation significantly reduced these compared to both groups. In males, both groups of $o b / o b$ mice had undetectable or unquantifiable ZO-1 values for most or all animals (responses smaller than the y-intercept of 
the standard curve). However, in females, ob/ob supplementation with CE reversed this effect and resulted in very high ZO-1 levels.

\subsection{Adiposity and inflammation biomarkers.}

Serum and hepatic triglyceride levels were measured, and these values are presented in Figure 5A and B and Supplementary Figure 5, respectively. Sex-specific effects of CE supplementation were observed in serum, but not hepatic, triglycerides. In males, serum triglycerides were slightly but non-significantly elevated in $o b / o b$ mice compared to WT controls, with no effect of CE supplementation. In females, serum triglycerides were slightly but non-significantly elevated in $o b / o b$ mice compared to WT controls (as in males), but CE supplementation resulted in substantial ( 15-fold) increases. Liver triglyceride levels were similar for males and females: triglycerides were slightly but non-significantly elevated in $o b / o b$ mice compared to WT controls and non-significantly blunted by CE supplementation (overall treatment effect was bordering on significance at $\mathrm{p}=0.07$ ). Canonical measures of inflammation (serum and hepatic TNF- $\alpha$, colonic COX-2) and liver damage (ALT) were measured, and these results are presented in Supplementary Figure 6. No significant differences were observed in these markers between groups due to the small $n$ size and high variability in one or more groups per assay.

Male

A

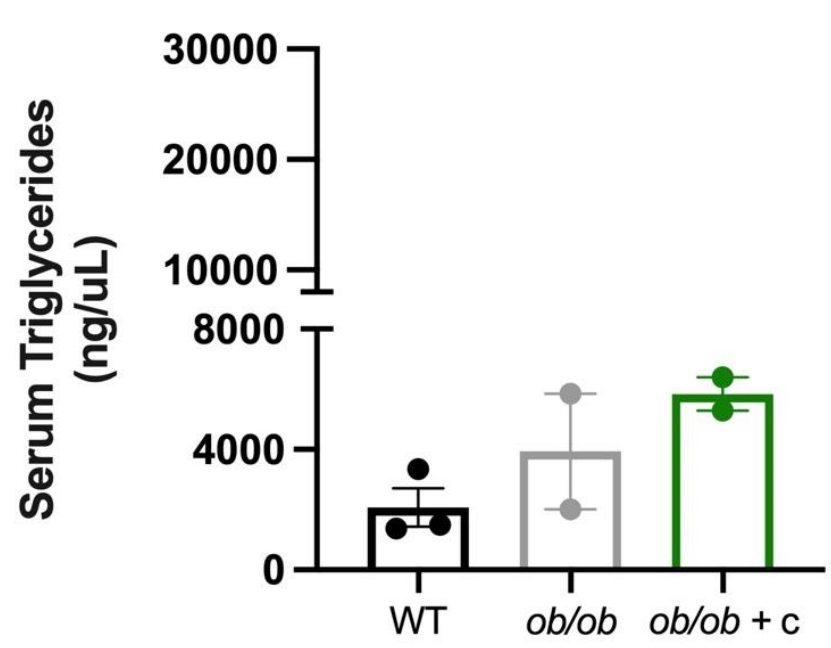

\section{Female}

B

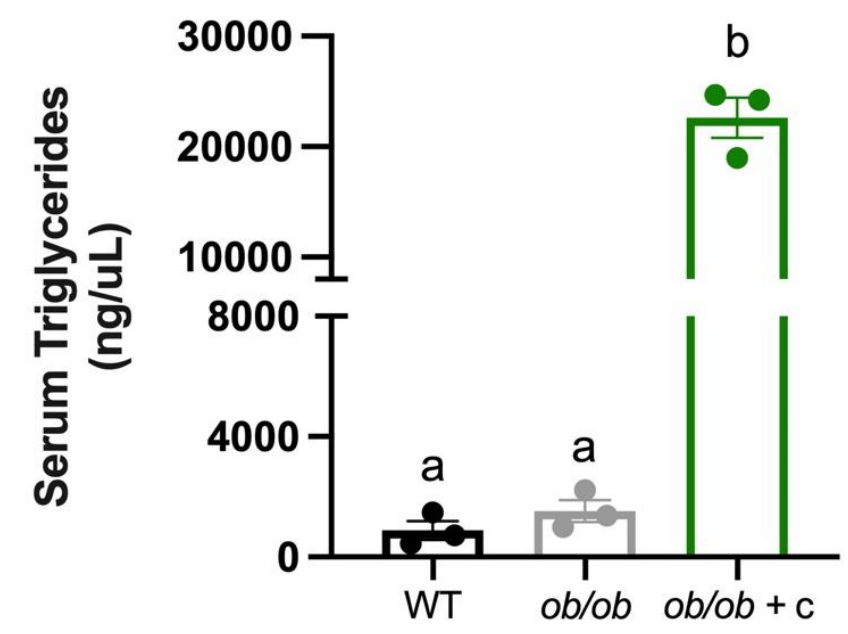

Figure 5. Serum triglyceride levels following 10 weeks of treatment for male $(\boldsymbol{A})$ and female $(\boldsymbol{B})$ mice. Values are presented as mean \pm SEM. Data were analyzed by 1-way ANOVA. If a significant treatment effect was detected, Tukey's post hoc test was performed to compare treatment means. Bars not sharing a common superscript letter are significantly different $(P<0.05)$.

\subsection{Hepatic markers of glycemic control.}

Hepatic levels of the gluconeogenesis enzyme PEPCK were quantified (Supplementary Figure 7). In males, there were no apparent differences in PEPCK levels. In females, due to high variability in $o b / o b$ control mice, there were also no differences in PEPCK levels.

\subsection{Pancreatic beta cell area}

Total beta cell area was measured by immunohistochemistry (insulin positive area) to determine if the $o b / o b$ mutation and/or CE feeding affected beta cell survival and is illustrated in Figure 6. In males, although not significant $(p=0.073)$, WT had slightly greater beta cell area overall compared to both $o b / o b$ and $o b / o b+c$ treatments (no effect of cocoa). In females, ob/ob controls had significantly reduced beta cell area compared to WT, but cocoa administration completely protected against beta cell loss. These observations are reiterated in the representative histological imaging of male (Figure 6A) and female (Figure 6C) pancreata 
A

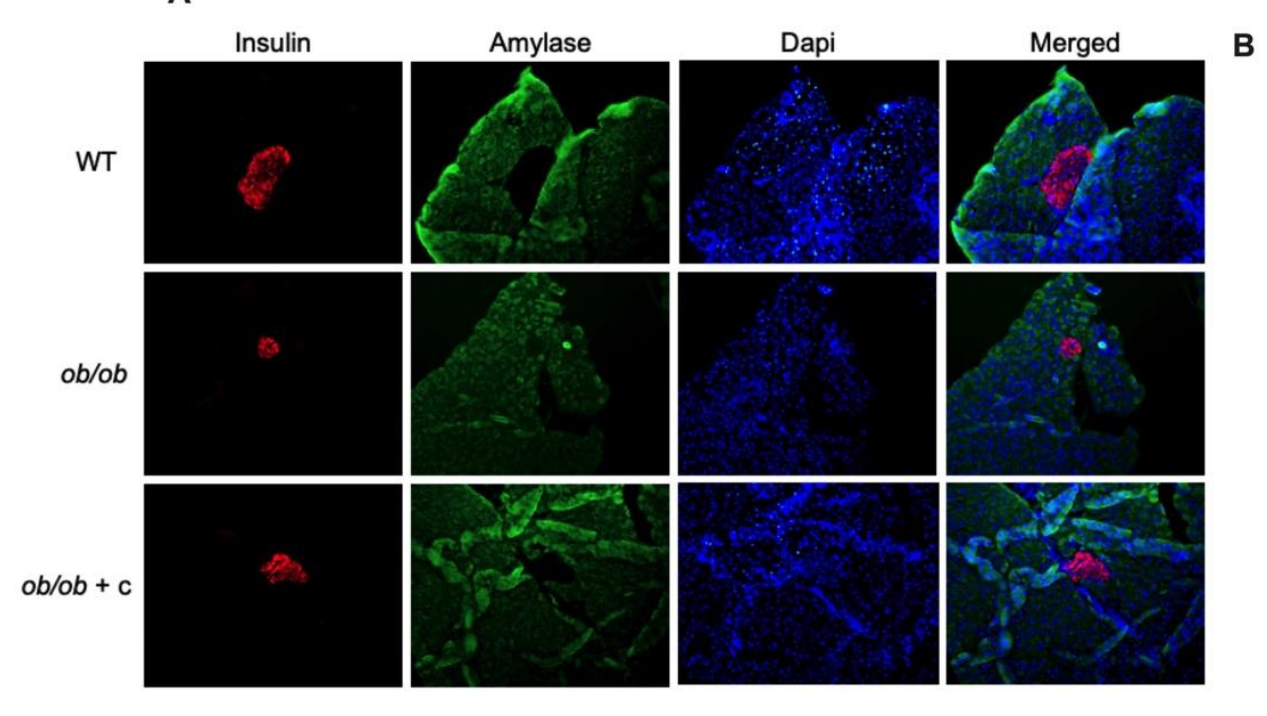

C

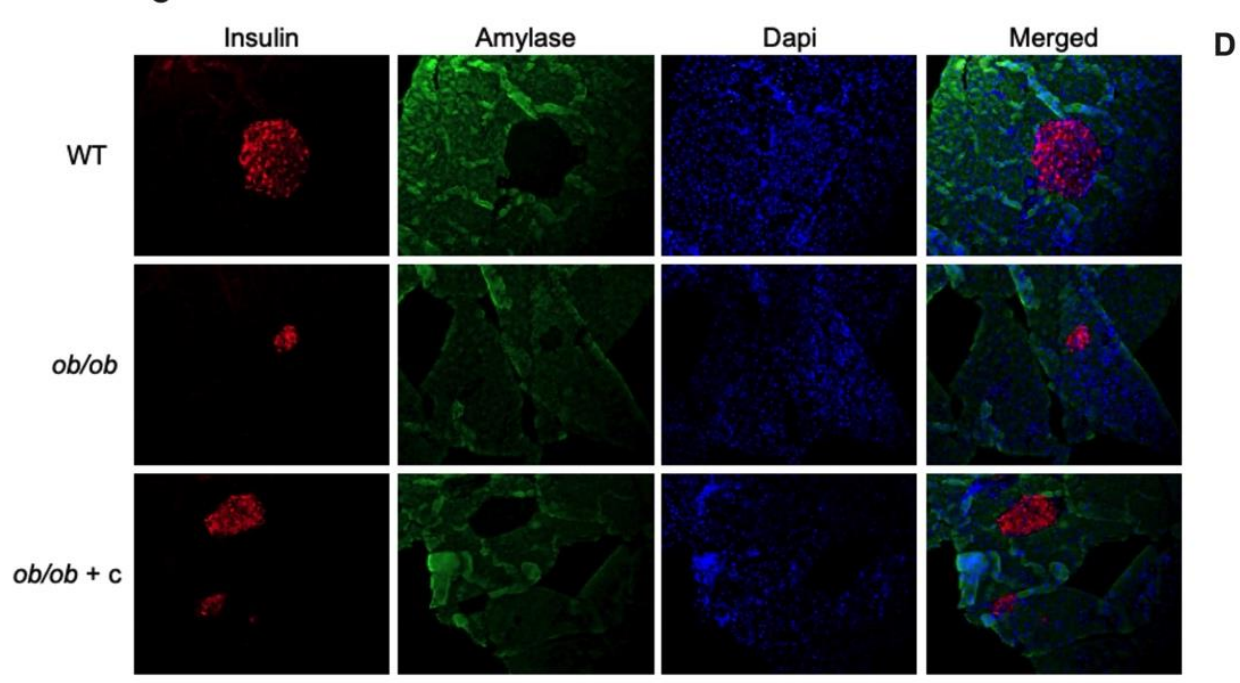

B

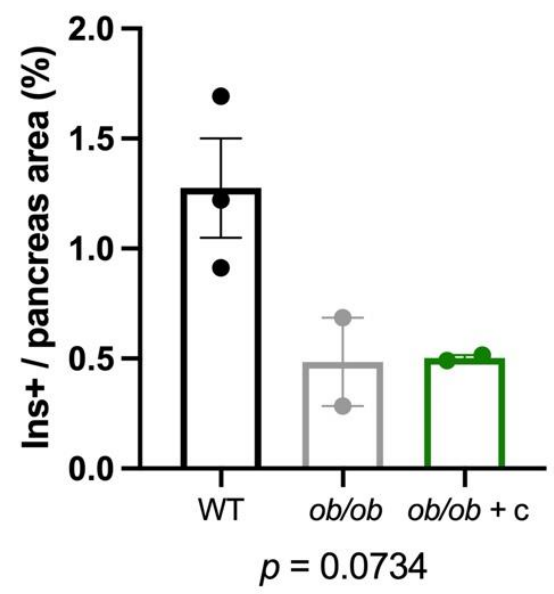

D

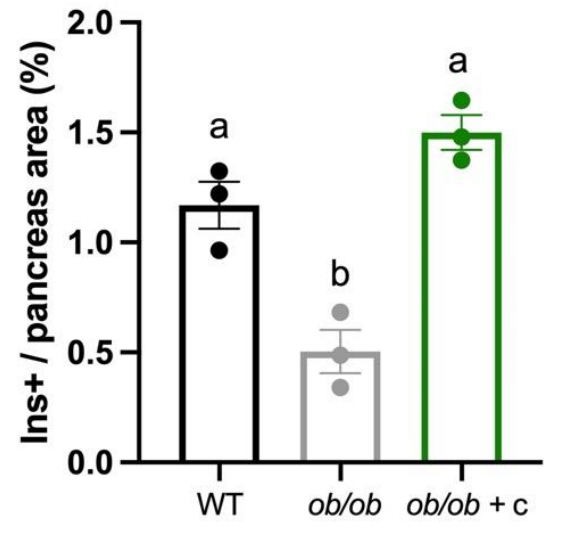

Figure 6. Representative histology images for pancreata (males: A, females: C). Total beta-cell area expressed as \% insulin positive/total pancreas area for males and females following 10 weeks of treatment (males: $\boldsymbol{B}$, females: $\boldsymbol{D}$ ). Images were captured at $20 x$ magnification and analyzed using cellSens and ImageJ software for five tissue section slides per animal. Values are presented as mean \pm SEM. Data were analyzed by 1-way ANOVA. If a significant treatment effect was detected, Tukey's post hoc test was performed to compare treatment means. Bars not sharing a common superscript letter are significantly different $(P<0.05)$.

\subsection{Circulating levels of estradiol (E2)}

Circulating levels of the major estrogen form $17 \beta$-estradiol were measured and are presented in Supplementary Figure 8. Although no significant differences were observed within male or female treatments, a trend of decreased levels was observed for both males and females supplemented with cocoa. As anticipated, female levels of estradiol were over 20X those of males.

\subsection{Animal survival.}

Due to rapid progression to severe hyperglycemia, the study was terminated earlier than planned. At the later stages of the study, veterinarian supervision was needed due to the rapidly declining health of multiple animals. At week 10 (mice were 14 weeks old), one male mouse (ob/ob control) was found deceased in its cage, and 3 female mice (all 3 from $o b / o b+c)$ were deemed to require euthanasia on humane grounds. Therefore, the study was terminated. This 
further demonstrates the aggressive phenotype as well as the potentially deleterious nature of cocoa in females in this context. It is worth noting that tissue and blood were not obtainable for the male mouse found deceased and are thus not included in the data and was unable to collect genotypic identification (Supplementary Table 1). This male mouse was consistently smaller than the other $o b / o b$ male mice in its cage, weighing $28.6 \mathrm{~g}$ at week 1 and reaching a max weight of $40.1 \mathrm{~g}$ at week 9 , compared to an average of $37.5 \mathrm{~g}$ at week 1 for the remaining two animals and reaching a max average weight of $55 \mathrm{~g}$ at week 10 . However, despite the consistent size difference, this mouse maintained consistent blood glucose values, both fasted and during challenges, to that of the other two animals in its cage.

\section{Discussion}

In this pilot study, we compared the effects of cocoa supplementation on accelerated progression of obesity-induced T2D in both male and female BTBR.Cg-Lepob/WiscJ mice. The ob/ob mutation on the BTBR background is an aggressive model of hyperglycemia, as evidence by our glucose data. To the best of our knowledge, this is the first study of any flavanol or flavanol-rich food in $o b / o b$ T2D mice on the BTBR background, as well as one of the few studies to simultaneously compare the effects of cocoa on both sexes. Within the realm of cocoa and its effect on inflammation, obesity, and associated disease pathologies, very few investigators utilize male and female mice simultaneously $[15,43]$. Despite the small sample size, the present results suggest significant differences in outcomes between male and female $o b / o b$ mice when fed a diet supplemented with flavanol-rich cocoa extract.

Sex differences between male and female mice emerged not only in established markers of T2D but also in physical observation and collection of weight gain over time. Although not statistically significant due to our small sample size, our data suggest that cocoa supplementation may have provided slight protection against obesity in male $o b / o b+$ c mice, yet females of the same treatment displayed similar weight gain over the 10 weeks as ob/ob control (Figure 1). Large variability was seen within the body weights of both male and female $o b / o b$ control mice, yet when cocoa supplementation is introduced, that variability was lost. We have previously reported that impacts of flavanol supplementation on body weight and glycemic control vary significantly between sexes in mice [44] but a larger sample size is needed to further understand this phenotypic variation as it has not been previously reported in BTBR $o b / o b$ males or females $[7,34,45,46]$.

The speed of T2D progression was notable in this study, as the cascade of hyperglycemia and hyperinsulinemia is accelerated when the $o b$ mutation is introduced on the BTBR background. It has been previously reported that BTBR $o b / o b$ mice are unable to maintain adequate insulin production, have impaired glucose tolerance, and are already insulin resistant at 4-6 weeks of age, in contrast to B6 ob/ob mice which are capable of compensating for insulin resistance [31]. In our study, fasting blood glucose at 5 weeks of age (week 1 GTT) was over $200 \mathrm{mg} / \mathrm{dL}$ and at 9 weeks of age (week 5 GTT) was between 400-550 mg/dL for male and female $o b / o b$ control mice (Figure 2A, B). Lan et al [7] reported similar fasting blood glucose levels for BTBR ob/ob mice and directly compared these mice to age-matched B6 ob/ob that display fasting blood glucose levels $<250 \mathrm{mg} / \mathrm{dL}$ at 9 weeks of age. Additionally, by 9 weeks of age, male and female BTBR $o b / o b$ mice exhibit extremely limited glucose clearance regardless of cocoa supplementation (Figures 2E, F). It must be noted that the maximum glucometer reading was $600 \mathrm{mg} / \mathrm{dL}$ therefore the true glucose level, and ultimately the variation/excursion of these animals was unable to be determined.

Cocoa supplementation appeared to have some acute effects, with improved glucose tolerance in both males and females at week 1 (Figures 2C, D). These acute benefits were more apparent in females than males, and observed only a few days after dietary treatment began. In males, CE appeared to worsen glycemic control at 5 weeks (Figure $2 \mathrm{E}$ ) and insulin sensitivity at 2 and 6 weeks (Figures 3C, E). Cocoa also appeared to provide females significant protection against acute insulin resistance compared to $o b / o b$ control (week 2), but this protection was lost by roughly the midpoint of the study (week 6, Figures 3C, E, D and F). Due to the extremely aggressive nature of T2D progression in BTBR $o b / o b$ mice, this benefit observed in females was not sustainable through nutritional prevention strategies. As mentioned above, BTBR $o b / o b$ mice are reported to be insulin resistant by 6 weeks of age, furthering our hypothesis that cocoa does in fact provide protection against initial disease progression specifically in female mice. A larger sample size and more frequent insulin challenges must be conducted in the future to delineate the role of cocoa flavanols in the protection of BTBR $o b / o b$ females against T2D progression.

These data also suggest that cocoa effects potentially reverse based on sex as T2D progresses. Cocoa treatment appeared to improve hyperglycemia-induced hyperinsulinemia in males but result in worsening of markers in females under fasting conditions (Figure 4). Whether this is due to changes in beta cell function and/or differences in insulin resistance is unknown, and further studies are needed to delineate the underlying mechanism(s). However, 
large (10-fold) differences in fasting insulin levels between males and females fed CE cannot be explained by differences in fasting blood glucose alone. Cocoa protected against beta cell loss in females (Figure 6) and together with fasting insulin expression, these results potentially indicate that in females, although CE did not provide relief against insulin resistance and hyperglycemia-induced hyperinsulinemia, it protected beta cell health and function. On the other hand, CE reduced insulin resistance and hyperglycemia-induced hyperinsulinemia in males, but that protection was not carried across to the beta cells. We previously reported that cocoa monomers and cocoa flavanol microbial metabolites promote beta cell stability and enhance/stimulate beta cell function in vitro $[23,47]$. These effects appear to translate in vivo in female mice supplemented with CE, but it remains unknown as to whether these effects are primarily because of hormonal differences or due to other underlying differences between sexes. If these effects are translatable to humans, cocoa may be useful at different stages of beta cell function and insulin resistance based on sex.

The impacts of both $o b / o b$ mutation and cocoa on gut barrier markers were unexpected, as obesity is typically associated with greater permeability and increased zonulin (a circulating regulator and marker of impaired tight junctions) [48]. The $o b / o b$ mutation caused a sex-specific loss of serum zonulin in males, which was partly ameliorated by cocoa (Supplementary Figure 4A, B). The opposite was seen in females, with CE decreasing levels of serum zonulin. ZO-1 results were opposite that of zonulin, as ob/ob mice had no detectable ZO-1, which was ameliorated to different degrees by cocoa in both sexes (Supplementary Figure 4C, D). Previous studies have shown that zonulin levels typically increase due to the $o b / o b$ mutation and T2D [49-51] and that dietary flavanol supplementation protects against ZO-1 delocalization and blunts the increase in circulating zonulin levels [52]. This suggests that cocoa may improve gut barrier function in obesity, with perhaps a greater effect in females.

We also observed that cocoa did not appear to affect fasting serum triglycerides in males but caused large increases in females, having significantly higher levels than female $o b / o b$ control (Figures 5 ). The mechanism for this difference, as well as its implications, are unknown as these differences were not reflected in body weight of male vs. female mice (Figure 1). The combination of elevated circulating insulin and serum triglycerides, particularly in female $o b / o b+c$ mice, can potentially be explained by more severe hyperglycemia and hyperinsulinemia. It has been thought that estrogen has the potential to confer protection against T2D progression through the prevention of beta cell failure, providing beneficial activities through a variety of pathways [34]. Figure 6 illustrates that CE supplementation preserved, and even elevated, beta cell survival in female $o b / o b$ mice, however this effect, along with the interaction of elevated serum insulin and triglycerides, is unlikely to be attributed to estrogen mediation as there were no significant differences observed in $17 \beta$-estradiol levels across female treatments (Supplementary Figure 8). The extreme worsening of hyperinsulinemia and apparent insulin resistance in females remains therefore unexplained. A recent study reported worsening glucose metabolism in a mice fed purified polyphenols diet-induced obese mouse model [53]. These data suggests that the potential anti-obesity and anti-diabetic benefits of cocoa may differ in mice based on T2D stage, biological sex, and/or hormonal status. Where these sex-specific effects are seen in humans remains to be established. Future studies should also capture the metabolic response in the liver, skeletal muscle, and adipose tissue to better understand these relationships between sex, beta cell function, and glucose homeostatic perturbation.

This experiment was designed as a pilot study, with the inherent limitation of a small sample size $(n=3)$ and a single cage per treatment. An additional limitation is the progressive nature of hyperglycemia in this model. Disease onset may have been impossible to significantly blunt in the long-term with a reasonable dose of dietary cocoa, however, these data provide insights into the potential effects of cocoa in the context of severe hyperglycemia. Despite these limitations, sex-specific effects of cocoa on hyperglycemia and related metabolic biomarkers were evident. Additionally, the dose of cocoa employed was elevated due to the overeating phenotype characteristic of the ob/ob mutation (as our diets were formulated based on food intake levels typically observed in WT mice). The effects observed, and differences between sexes, may therefore be partly attributable to this high dose and future research is needed using more translatable doses. There are also significant challenges in working with this model. First and foremost, the cost for both heterozygotes and homozygotes of the $o b$ mutation on the BTBR background is very high, and therefore, availability is significantly limited compared to mice on the B6 background (on which the $o b$ mutation is typically used). The lead times for delivery were also long or unknown and age availability was limited, even from Jackson. Thus, the cost and availability of these mice inherently restrict the experiments that can be performed, consequently leading to the small sample size for this pilot study. However, given the preliminary findings, larger studies with more statistical power are warranted to further evaluate these findings. 
The findings of this pilot study, along with other recent studies from our group and others, highlight the need to factor in sex-specific disease biology and treatment effects when studying interventions in pre-clinical animal and human studies [44,54]. Too often, pre-clinical animal studies of a single strain and/or sex are utilized to evaluate the potential for a dietary bioactive to prevent or ameliorate chronic disease [55,56]. Studies should be performed to elucidate whether sex differences are primarily due to hormonal differences [57-59] as well as further elucidate the role that genetic background plays in disease outcome. We previously reported that the anti-obesity and anti-diabetic activity of quercetin was highly dependent on genetic background in mice [44]. It is unknown whether the sex-specific effects of cocoa in the present study are limited to mice with the $o b / o b$ mutation or the BTBR genetic background. These findings will need to be evaluated in the context of multiple preclinical mouse models of T2D (DIO, $d b / d b, \mathrm{HF}+$ STZ, etc.) at other doses. If the results are generalizable across strains, human work is warranted. If the results are specific to narrow genetic contexts, further studies can be performed to identify the genetic loci responsible for such effects. These results could then be applicable to human orthologues for potential personalized nutrition approaches targeting humans with specific genetic characteristics [60]. Finally, the sex-dependence of the effects of various cocoa components should be evaluated. Future studies are needed in both males and females, using multiple complementary preclinical models of obesity and hyperglycemia (diet-induced C57BL/6J, ob/ob, etc.) to further interrogate sexspecific effects of cocoa at various stages of hyperglycemia.

\section{Materials and Methods}

4.1 Chemical standards and reagents. LC-MS grade acetonitrile and methanol were obtained from Thermo-Fisher Scientific (Waltham, MA). Glacial acetic acid and acetone were obtained from VWR (Radnor, PA). Authentic standards of (-)-epicatechin, $( \pm$ )-catechin, and procyanidin B2 were obtained from ChromaDex (Irving, CA). Folin-Ciocalteu reagent and 4-dimethylaminocinnamaldehyde (DMAC) were obtained from Sigma-Aldrich (St. Louis, MO). Solvents were ACS grade or higher.

\subsection{Cocoa flavanol-rich extract production and characterization. A flavanol-rich cocoa extract (CE) was prepared as de-} scribed previously [61]. Briefly, commercially available non-alkalized natural cocoa powder (The Hershey Co., Hershey, PA) was defatted three times by dispersion in hexane (1:3.75 cocoa:hexane), incubated for 10 min with sonication, centrifugation $(5 \mathrm{~min}, 5000 \times \mathrm{g})$ and discarding the supernatant. Residual hexane was evaporated at room temperature. Flavanols were extracted from defatted cocoa by dispersion in extraction solution (70:28:2 acetone:water:acetic acid, v/v/v; 1:3.75 defatted cocoa:extraction solution), incubated for $10 \mathrm{~min}$ with sonication, centrifugation ( $5 \mathrm{~min}$, $5000 \times g)$ and collecting the supernatant. This process was repeated three times, supernatants were pooled, and solvent evaporated under rotary evaporation $\left(40-45^{\circ} \mathrm{C}\right)$. The remaining $\mathrm{CE}$ was freeze dried and stored at $-80^{\circ} \mathrm{C}$. CE was analyzed by the Folin-Ciocalteu and 4-dimethylaminocinnamaldehyde (DMAC) methods as described previously $[61,62]$ to approximate total polyphenols and total procyanidins, respectively. CE was thiolyzed with benzyl mercaptan and the resulting products quantified by UPLC-MS/MS as described previously $[36,61]$ to determine flavanol mean degree of polymerization (mDP). CE was also analyzed by UPLC-MS/MS as previously described [63] to measure monomeric flavanols through decameric procyanidins. Methodological details for characterization procedures are found in Supplementary Material.

4.3 Animals. Approval for this study was obtained from the Animal Care and Use Committee at the David H. Murdock Research Institute (\#20-011). Four-week-old BTBR.Cg-Lepob/WiscJ mice (stock \# 004824) were obtained from Jackson (Bar Harbor, ME): N=12 males and 12 females homozygous for the Lepob mutation (ob/ob), and N=6 male and 6 female wild-type (WT) controls. Mice were acclimatized to the facility for $4 \mathrm{~d}$ before cage randomization to experimental diets. Mice were housed under standard laboratory conditions (12 h light/dark cycle, 30-70\% relative humidity, $20-26^{\circ} \mathrm{C}, 3 \mathrm{mice} / \mathrm{cage}$ ) on aspen bedding. All animals were allowed access to food and water ad libitum except where otherwise specified.

4.4 Dietary treatments. All mice were fed the $10 \%$ fat, $7 \%$ sucrose control diet from the Diet-Induced Obesity (DIO) series (D12450J, Research Diets, New Brunswick, NJ) alone or supplemented with $0.8 \%$ CE by weight (8000 mg CE $/ \mathrm{kg}$ diet). Diet formulations are shown in Table 2. This dose was selected based on previous studies[41,42] demonstrating that $8 \%$ cocoa powder in the diet blunted diet-induced obesity without altering food intake in WT mice. Historically, 
our yield of CE from cocoa powder has been $\sim 10 \% \mathrm{w} / \mathrm{w}$. Therefore, $0.8 \% \mathrm{CE}$ in the diet provides cocoa flavanol content similar to $8 \%$ cocoa powder in the diet. Based on an estimated extraction yield of $10 \%$ from cocoa powder and estimated food intake of $\sim 0.1 \mathrm{~kg}$ diet $/ \mathrm{kg}$ body weight/day in WT mice, this dose was designed to provide $\sim 800 \mathrm{mg}$ $\mathrm{CE} / \mathrm{kg}$ body weight/day (equivalent to $\sim 8000 \mathrm{mg}$ cocoa powder/kg body weight/day, i.e. $8 \%$ cocoa) to mice eating normal amounts of food (as we had not previously worked with $o b / o b$ or BTBR mice). Based on body surface area conversion [64], this dose corresponds to $65 \mathrm{mg} \mathrm{CE} / \mathrm{kg}$ body weight/day ( $650 \mathrm{mg}$ cocoa powder/kg body weight/day) in adult humans. For a $60 \mathrm{~kg}$ individual, this would equate to $3900 \mathrm{mg}$ CE or $39 \mathrm{~g}$ cocoa powder. This equates to roughly 8 doses of cocoa powder ( $5 \mathrm{~g} /$ dose) per day. The treatment groups for each sex were as follows: WT, ob/ob, ob/ob+cocoa extract $(o b / o b+c)(n=3 /$ sex/group $)$. Diet treatments were started on the first day of week 1.

Table 2. Mouse diet formulations

\begin{tabular}{|c|c|c|c|c|}
\hline \multirow[b]{2}{*}{ Macronutrient } & \multicolumn{2}{|c|}{ Control diet } & \multicolumn{2}{|c|}{ Cocoa extract diet } \\
\hline & $\begin{array}{l}\% \\
\text { (g) } \\
\end{array}$ & $\begin{array}{c}\% \\
\text { (kcal) } \\
\end{array}$ & $\begin{array}{l}\% \\
(\mathrm{~g}) \\
\end{array}$ & $\begin{array}{c}\% \\
\text { (kcal) } \\
\end{array}$ \\
\hline Protein & 19 & 20 & 19 & 20 \\
\hline Carbohydrate & 67 & 70 & 67 & 70 \\
\hline Fat & 4 & 10 & 4 & 10 \\
\hline Total (\%) & & 100 & & 100 \\
\hline $\mathrm{kcal} / \mathrm{g}$ & 3.8 & & 3.8 & \\
\hline Ingredient & $\mathrm{g}$ & kcal & g & kcal \\
\hline Casein & 200 & 800 & 200 & 800 \\
\hline L-Cystine & 3 & 12 & 3 & 12 \\
\hline Corn Starch & 506.2 & 2025 & 506.2 & 2025 \\
\hline Maltodextrin 10 & 125 & 500 & 125 & 500 \\
\hline Sucrose & 68.8 & 275 & 68.8 & 275 \\
\hline Cellulose, BW200 & 50 & 0 & 50 & 0 \\
\hline Soybean Oil & 25 & 225 & 25 & 225 \\
\hline Lard & 20 & 180 & 20 & 180 \\
\hline Mineral Mix S10026 & 10 & 0 & 10 & 0 \\
\hline Dicalcium Phosphate & 13 & 0 & 13 & 0 \\
\hline Calcium Carbonate & 5.5 & 0 & 5.5 & 0 \\
\hline Potassium Citrate, $1 \mathrm{H} 2 \mathrm{O}$ & 16.5 & 0 & 16.5 & 0 \\
\hline Vitamin Mix V10001 & 10 & 40 & 10 & 40 \\
\hline Choline Bitartrate & 2 & 0 & 2 & 0 \\
\hline Cocoa Extract & 0 & 0 & 8.51 & 0 \\
\hline FD\&C Yellow Dye \#5 & 0.04 & 0 & 0 & 0 \\
\hline FD\&C Red Dye \#40 & 0 & 0 & 0.05 & 0 \\
\hline FD\&C Blue Dye \#1 & 0.01 & 0 & 0 & 0 \\
\hline Total & 1055.05 & 4057 & 1063.56 & 4057 \\
\hline
\end{tabular}


4.5 Body weight and food intake. Body weight and food intake (measured by difference between food provided and food remaining) were measured weekly.

4.6 Fasting blood glucose and insulin measurement. Glucose tolerance tests (GTTs) were performed during weeks 1 and 5. Mice were fasted for $6 \mathrm{~h}$, weighed, and glucose administered i.p. using tuberculin syringes containing a $20 \%$ dextrose solution (USP-grade, Hospira, Inc., Lake Forest, IL) in sterile saline to provide $2 \mathrm{~g}$ glucose/kg body mass. Blood droplets were collected from the tail vein at 0 (pre-administration), 30, 60, 90, and 120 min after injection. Blood glucose was measured using OneTouch Ultra Blue glucose test strips (LifeScan, Inc., Milpitas, CA) and a glucometer. Excursion from baseline, maximal glucose level $\left(\mathrm{C}_{\max }\right)$, and area under the curve (AUC) were measured for week 5 GTT. Insulin tolerance tests (ITTs) were performed at weeks 2 and 6 . Mice were fasted for $4 \mathrm{~h}$, weighed, and provided insulin (Humulin R, Cardinal Health, Dublin, $\mathrm{OH}$ ) i.p. at $0.65 \mathrm{U} / \mathrm{kg}$ body mass in sterile saline via tuberculin syringes. Blood was collected at $0,15,30,45$, and $60 \mathrm{~min}$ and glucose measured as described above. The upper limit of the glucometer was $600 \mathrm{mg} / \mathrm{dL}$; values above that were noted as such, and $600 \mathrm{mg} / \mathrm{dL}$ was used for calculation purposes. AUC was calculated for week 2 and 6 ITT.

\subsection{Euthanasia and tissue collection}

Upon study completion, animals were fasted for $12 \mathrm{~h}$ and euthanized by $\mathrm{CO}_{2}$, followed by cervical dislocation, per AVMA guidelines. Blood was collected via cardiac puncture. Following blood collection, the abdomen was opened ventrally and samples collected from the colon, cecum, small intestine, pancreas, liver. Samples were stored in RIPA buffer (Thermo Fisher, Waltham, MA) for protein analyses. Pancreas and liver samples were preserved in cassettes immersed initially in 10\% neutral buffered formalin solution (Thermo Fisher) overnight and then stored in $70 \%$ ethanol. Blood was collected in MiniCollect Serum Tubes (Greiner Bio-One, Monroe, NC) and clotted at room temperature for $30 \mathrm{~min}$. Serum was prepared by centrifuging $(2000 \times g, 10 \mathrm{~min})$. Samples were stored at $-80^{\circ} \mathrm{C}$ prior to analysis.

4.8 Blood biomarkers. Fasting blood insulin was quantified using a mouse ultrasensitive insulin ELISA kit with the 0$12.8 \mathrm{ng} / \mathrm{mL}$ "wide" standard curve range option (90080, Crystal Chem, Elk Grove Village, IL). Fasting blood triglycerides were quantified by a Triglyceride Quantification Colorimetric/Fluorometric Kit (MAK266-1KT, Sigma, St. Louis, MO). Zonulin, a circulating regulator of intestinal permeability, was measured by ELISA (MBS2603528, MyBiosource.com, San Diego, CA). Alanine aminotransferase (ALT) and TNF- $\alpha$, markers of liver damage and systemic inflammation, respectively, were quantified by ELISA (MBS264717 and MBS825075, MyBiosource.com). Circulating serum levels of estradiol (E2 or 17 $\beta$-estradiol) were measured by ELISA (MBS843418, MyBiosource.com).

4.9 Intestinal biomarkers. Colon and cecum samples were prepared for analysis by bead beating and centrifugation. Half of the collected tissue was combined with RIPA buffer, bead beat for 20 min, incubated with sonication for 5 min, followed by centrifugation $(17000 \times g, 5 \mathrm{~min})$. Supernatants were stored at $-80^{\circ} \mathrm{C}$ until assayed for various markers by ELISA: ZO-1 (MBS2603798, MyBiosource.com), COX-2 (MBS269104, MyBiosource.com). Total protein was quantified in homogenates using the Pierce BCA Protein Assay Kit (ThermoScientific, Catalog No. 23225, 23227).

4.10 Liver biomarkers. Liver samples were prepared by bead beating and centrifugation. Approximately 200 mg sample was combined with RIPA buffer, bead beat for $5 \mathrm{~min}$, incubated with sonication for $5 \mathrm{~min}$, followed by centrifugation $(17000 \times g, 5 \mathrm{~min})$. Supernatants were stored at $-80^{\circ} \mathrm{C}$ until analyzed. Phosphoenolpyruvate carboxykinase (PEPCK), a marker of gluconeogenesis, was quantified by ELISA (MBS3805602, MyBiosource.com). Protein was quantified in homogenates as described above.

4.11 Histology. Pancreata were embedded in paraffin and five sections were cut from each animal. Sections were deparaffinized in xylene and rehydrated using a graded ethanol series. Antigen retrieval was performed using a sodium citrate buffer (Vector, H-3300-250) according to manufacturer's protocol. For insulin staining, slides were incubated overnight with guinea pig anti-insulin antibody (Fitzgerald, 20-IP35) followed by detection with an AlexaFluor 555conjugated (red) goat-anti guinea pig secondary antibody (ThermoFisher, A-21435). For total tissue section area, slides were incubated overnight with rabbit $\alpha$-Amylase (Sigma, A8273), followed by detection with an AlexaFluor 488-conjugated (green) goat anti-rabbit secondary antibody (ThermoFisher, A-32731). Slides were counterstained with DAPI. Images were captured at 20x magnification and analyzed using cellSens and ImageJ software for five tissue section 
slides per animal. $\beta$-cell area was determined as the total insulin-stained area divided by the total pancreatic tissue area (amylase and insulin-stained area) per slide.

4.12 Animal genotyping. Formalin fixed liver tissue was used to genotype each animal to confirm the presence or absence of the homozygous $o b / o b$ mutation. Samples were sent to Transnetyx (Cordova, TN) and results reported for all samples collected.

4.13 Statistical analysis. Data were analyzed using GraphPad Prism v9.1.2 (La Jolla, CA, USA). Outliers were removed by Dixon's q-test at $\alpha=0.05$. When values were missing due to removal of outliers, missed sample, or animal death, a mixed-effects model was used. When initial one-way or two-way ANOVA indicated a significant overall treatment effect $(\mathrm{P}<0.05)$, individual means were compared using Tukey's post-hoc test or Sidak's post-hoc test to control for multiple comparisons $(\alpha=0.05)$. Power analyses were performed using G*Power (Version 3.1.9.3, Heinrich-HeineUniversität, Düsseldorf, Germany).

\section{Conclusions}

Cocoa supplementation at high doses appears to have sex-specific effects in the context of severe, progressive hyperglycemia. Cocoa appears to acutely prevent against some measures of glucose intolerance in both sexes, and insulin resistance in females, in the short term. Chronic supplementation appears to ameliorate obesity-induced hyperinsulinemia in males and worsen hyperlipidemia and obesity-induced hyperinsulinemia, yet preserve and enhance beta cell survival, in females. Effects on gut barrier function also appear sex-specific, although the implications of these differences are unclear. The underlying mechanisms of these sex differences, as well as their implications for human nutrition, warrant further study. The present pilot data will inform additional experiments with larger sample sizes to further elucidate sex-specific effects of cocoa. Whether these sex-specific effects are observed in other preclinical models of T2D in addition to the present BTBR $o b / o b$ model remains to be established. OVX and estrogen inhibitor/replacement studies are now justified to determine the hormonal basis for such differences. If sex differences are apparent in subsequent studies, clinical studies will be warranted to establish whether these differences are relevant in humans and in what contexts. There exists the possibility that sex-specific differences may need to be taken into consideration when designing intervention strategies to prevent or ameliorate T2D at the individual and population levels.

Supplementary Materials: The following are available online at www.mdpi.com/xxx/s1, supplemental methodological details, Supplementary Table 1. Mouse genotyping results; Supplementary Figure 1. Absolute weights; Supplementary Figure 2. Cocoa extract intake; Supplementary Figure 3. Additional glucose tolerance test measures; Supplementary Figure 4. Serum zonulin and colonic zona occludens-1 levels; Supplementary Figure 5. Hepatic triglyceride levels; Supplementary Figure 6. Serum TNF- $\alpha$, hepatic TNF- $\alpha$, colonic COX-2, and serum ALT levels; Supplementary Figure 7. Hepatic phosphoenolpyruvate carboxykinase levels; Supplementary Figure 8. Serum estradiol levels.

Author Contributions: Conceptualization: MGF, CDK, JST and APN; Methodology; KCR, LIC, JAH, MGF, CDK, JST and APN; Software: N/A.; Validation: MGF, CDK, JST and APN; Formal Analysis: KCR, JAH, JST, APN; Investigation: KCR, LIC, JAH, JST and APN; Resources: MGF, CDK, JST and APN; Data Curation: JST and APN; Writing - Original Draft Preparation: KCR; Writing Review \& Editing: KCR, LIC, JAH, MGF, CDK, JST and APN; Visualization: KCR and JAH; Supervision: JST and APN; Project Administration: JST and APN; Funding Acquisition: MGF, CDK, JST and APN

Funding: This work was funded by the US Department of Agriculture by NIFA AFRI foundational grant 2020-67017-30846 (APN, JST, CDK, and MGF).

Institutional Review Board Statement: The study was conducted according to the guidelines of the Declaration of Helsinki, and proved by Animal Care and Use Committee at the David H. Murdock Research Institute (\#20-011).

Informed Consent Statement: Not applicable.

Data Availability Statement: The data presented in this study are available on request from the corresponding author.

Acknowledgements. The authors wish to acknowledge Dr. Glicerio Ignacio, DVM (attending veterinarian) and Mr. Daniel Peralta (facility manager) of the David H. Murdock Research Institute for their invaluable contributions to animal care, husbandry, and 
phenotype monitoring, as well as Lauren Essenmacher for her contribution to animal husbandry and blood glucose testing and Lyric Ramsue for her assistance with ELISA assays.

Conflicts of Interest: The authors declare no conflicts of interest.

Sample Availability: Samples are not available.

\section{References}

1. Skyler, J.S.; Bakris, G.L.; Bonifacio, E.; Darsow, T.; Eckel, R.H.; Groop, L.; Groop, P.-H.; Handelsman, Y.; Insel, R.A.; Mathieu, C.; et al. Differentiation of Diabetes by Pathophysiology, Natural History, and Prognosis. Diabetes 2017, 66, 241-255, doi:10.2337/db16-0806.

2. Alejandro, E.U.; Gregg, B.; Blandino-Rosano, M.; Cras-Méneur, C.; Bernal-Mizrachi, E. Natural History of $\beta$-Cell Adaptation and Failure in Type 2 Diabetes. Mol. Aspects Med. 2015, 42, 19-41, doi:10.1016/j.mam.2014.12.002.

3. Prentki, M.; Nolan, C.J. Islet $\beta$ Cell Failure in Type 2 Diabetes. J. Clin. Invest. 2006, 116, 1802-1812, doi:10.1172/JCI29103.

4. Hudish, L.I.; Reusch, J.E.B.; Sussel, L. $\beta$ Cell Dysfunction during Progression of Metabolic Syndrome to Type 2 Diabetes. J. Clin. Invest. 129, 4001-4008, doi:10.1172/JCI129188.

5. IDF Diabetes Atlas 9th Edition 2019 Available online: https://diabetesatlas.org/en/ (accessed on 13 March 2020).

6. Cerf, M.E. Beta Cell Dysfunction and Insulin Resistance. Front. Endocrinol. 2013, 4, 37, doi:10.3389/fendo.2013.00037.

7. Lan, H.; Rabaglia, M.E.; Stoehr, J.P.; Nadler, S.T.; Schueler, K.L.; Zou, F.; Yandell, B.S.; Attie, A.D. Gene Expression Profiles of Nondiabetic and Diabetic Obese Mice Suggest a Role of Hepatic Lipogenic Capacity in Diabetes Susceptibility. Diabetes 2003, 52, 688-700, doi:10.2337/diabetes.52.3.688.

8. Cedó, L.; Castell-Auví, A.; Pallarès, V.; Blay, M.; Ardévol, A.; Arola, L.; Pinent, M. Grape Seed Procyanidin Extract Modulates Proliferation and Apoptosis of Pancreatic Beta-Cells. Food Chem. 2013, 138, 524-530.

9. Pan, M.-H.; Lai, C.-S.; Ho, C.-T. Anti-Inflammatory Activity of Natural Dietary Flavonoids. Food Funct. 2010, 1, 15-31.

10. Vinayagam, R.; Xu, B. Antidiabetic Properties of Dietary Flavonoids: A Cellular Mechanism Review. Nutr. Metab. 2015, 12, 1-20.

11. Babu, P.V.A.; Liu, D.; Gilbert, E.R. Recent Advances in Understanding the Anti-Diabetic Actions of Dietary Flavonoids. J. Nutr. Biochem. 2013, 24, 1777-1789.

12. Schinella, G.; Mosca, S.; Cienfuegos-Jovellanos, E.; Pasamar, M.A.; Muguerza, B.; Ramon, D.; Rios, J.L. Antioxidant Properties of Polyphenol-Rich Cocoa Products Industrially Processed. Food Res. Int. 2010, 43, $1614-1623$.

13. Oracz, J.; Zyzelewicz, D.; Nebesny, E. The Content of Polyphenolic Compounds in Cocoa Beans (Theobroma Cacao L.), Depending on Variety, Growing Region, and Processing Operations: A Review. Crit. Rev. Food Sci. Nutr. 2015, 55, 1176-1192, doi:10.1080/10408398.2012.686934.

14. Andújar, I.; Recio, M.C.; Giner, R.M.; Ríos, J.L. Cocoa Polyphenols and Their Potential Benefits for Human Health. Oxid. Med. Cell. Longev. 2012, 2012.

15. Ramos, S.; Martín, M.A.; Goya, L. Effects of Cocoa Antioxidants in Type 2 Diabetes Mellitus. Antioxidants 2017, 6, 84, doi:10.3390/antiox6040084.

16. Martín, M.Á.; Fernández-Millán, E.; Ramos, S.; Bravo, L.; Goya, L. Cocoa Flavonoid Epicatechin Protects Pancreatic Beta Cell Viability and Function against Oxidative Stress. Mol. Nutr. Food Res. 2014, 58, 447-456, doi:10.1002/mnfr.201300291.

17. Martín, M.Á.; Cordero-Herrera, I.; Bravo, L.; Ramos, S.; Goya, L. Cocoa Flavanols Show Beneficial Effects in Cultured Pancreatic Beta Cells and Liver Cells to Prevent the Onset of Type 2 Diabetes. Food Res. Int. 2014, 63, 400408, doi:10.1016/j.foodres.2014.05.006. 
18. Kose, T.; Vera-Aviles, M.; Sharp, P.A.; Latunde-Dada, G.O. Curcumin and (-)- Epigallocatechin-3-Gallate Protect Murine MIN6 Pancreatic Beta-Cells against Iron Toxicity and Erastin-Induced Ferroptosis. Pharmaceuticals 2019, 12, 26, doi:10.3390/ph12010026.

19. Sun, P.; Wang, T.; Chen, L.; Yu, B.; Jia, Q.; Chen, K.; Fan, H.; Li, Y.; Wang, H. Trimer Procyanidin Oligomers Contribute to the Protective Effects of Cinnamon Extracts on Pancreatic $\beta$-Cells in Vitro. Acta Pharmacol. Sin. 2016, 37, 1083-1090, doi:10.1038/aps.2016.29.

20. Fernández-Millán, E.; Cordero-Herrera, I.; Ramos, S.; Escrivá, F.; Alvarez, C.; Goya, L.; Martín, M.A. Cocoa-rich Diet Attenuates Beta Cell Mass Loss and Function in Young Zucker Diabetic Fatty Rats by Preventing Oxidative Stress and Beta Cell Apoptosis. Mol. Nutr. Food Res. 2015, 59, 820-824.

21. YAMASHITA, Y.; OKABE, M.; NATSUME, M.; ASHIDA, H. Cinnamtannin A2, a Tetrameric Procyanidin, Increases GLP-1 and Insulin Secretion in Mice. Biosci. Biotechnol. Biochem. 2013, 77, 888-891, doi:10.1271/bbb.130095.

22. Castell-Auví, A.; Cedó, L.; Pallarès, V.; Blay, M.; Pinent, M.; Ardévol, A. Grape Seed Procyanidins Improve $\beta$-Cell Functionality under Lipotoxic Conditions Due to Their Lipid-Lowering Effect. J. Nutr. Biochem. 2013, 24, 948-953, doi:10.1016/j.jnutbio.2012.06.015.

23. Rowley IV, T.J.; Bitner, B.F.; Ray, J.D.; Lathen, D.R.; Smithson, A.T.; Dallon, B.W.; Plowman, C.J.; Bikman, B.T.; Hansen, J.M.; Dorenkott, M.R. Monomeric Cocoa Catechins Enhance $\beta$-Cell Function by Increasing Mitochondrial Respiration. J. Nutr. Biochem. 2017, 49, 30-41.

24. Kord-Varkaneh, H.; Ghaedi, E.; Nazary-Vanani, A.; Mohammadi, H.; Shab-Bidar, S. Does Cocoa/Dark Chocolate Supplementation Have Favorable Effect on Body Weight, Body Mass Index and Waist Circumference? A Systematic Review, Meta-Analysis and Dose-Response of Randomized Clinical Trials. Crit. Rev. Food Sci. Nutr. 2019, 59, 2349-2362, doi:10.1080/10408398.2018.1451820.

25. Sun, Y.; Zimmermann, D.; Castro, C.A.D.; Actis-Goretta, L. Dose-Response Relationship between Cocoa Flavanols and Human Endothelial Function: A Systematic Review and Meta-Analysis of Randomized Trials. Food Funct. 2019, 10, 6322-6330, doi:10.1039/C9FO01747J.

26. Yuan, S.; Li, X.; Jin, Y.; Lu, J. Chocolate Consumption and Risk of Coronary Heart Disease, Stroke, and Diabetes: A Meta-Analysis of Prospective Studies. Nutrients 2017, 9, 688, doi:10.3390/nu9070688.

27. Dicks, L.; Kirch, N.; Gronwald, D.; Wernken, K.; Zimmermann, B.F.; Helfrich, H.-P.; Ellinger, S. Regular Intake of a Usual Serving Size of Flavanol-Rich Cocoa Powder Does Not Affect Cardiometabolic Parameters in Stably Treated Patients with Type 2 Diabetes and Hypertension-A Double-Blinded, Randomized, Placebo-Controlled Trial. Nutrients 2018, 10, 1435, doi:10.3390/nu10101435.

28. Shah, S.R.; Alweis, R.; Najim, N.I.; Dharani, A.M.; Jangda, M.A.; Shahid, M.; Kazi, A.N.; Shah, S.A. Use of Dark Chocolate for Diabetic Patients: A Review of the Literature and Current Evidence. J. Community Hosp. Intern. Med. Perspect. 2017, 7, 218-221, doi:10.1080/20009666.2017.1361293.

29. Stoehr, J.P.; Byers, J.E.; Clee, S.M.; Lan, H.; Boronenkov, I.V.; Schueler, K.L.; Yandell, B.S.; Attie, A.D. Identification of Major Quantitative Trait Loci Controlling Body Weight Variation in Ob/Ob Mice. Diabetes 2004, 53, 245-249, doi:10.2337/diabetes.53.1.245.

30. King, A.J. The Use of Animal Models in Diabetes Research. Br. J. Pharmacol. 2012, 166, 877-894.

31. Clee, S.M.; Nadler, S.T.; Attie, A.D. Genetic and Genomic Studies of the BTBR Ob/Ob Mouse Model of Type 2 Diabetes. Am. J. Ther. 2005, 12, 491-498.

32. Ranheim, T.; Dumke, C.; Schueler, K.L.; Cartee, G.D.; Attie, A.D. Interaction between BTBR and C57BL/6J Genomes Produces an Insulin Resistance Syndrome in (BTBR× C57BL/6J) F1 Mice. Arterioscler. Thromb. Vasc. Biol. $1997,17,3286-3293$. 
33. Stoehr, J.P.; Nadler, S.T.; Schueler, K.L.; Rabaglia, M.E.; Yandell, B.S.; Metz, S.A.; Attie, A.D. Genetic Obesity Unmasks Nonlinear Interactions between Murine Type 2 Diabetes Susceptibility Loci. Diabetes 2000, 49, 1946-1954, doi:10.2337/diabetes.49.11.1946.

34. O’Brien, P.D.; Hur, J.; Hayes, J.M.; Backus, C.; Sakowski, S.A.; Feldman, E.L. BTBR Ob/Ob Mice as a Novel Diabetic Neuropathy Model: Neurological Characterization and Gene Expression Analyses. Neurobiol. Dis. 2015, 73, 348355, doi:10.1016/j.nbd.2014.10.015.

35. Zhao, E.; Keller, M.P.; Rabaglia, M.E.; Oler, A.T.; Stapleton, D.S.; Schueler, K.L.; Neto, E.C.; Moon, J.Y.; Wang, P.; Wang, I.-M.; et al. Obesity and Genetics Regulate MicroRNAs in Islets, Liver, and Adipose of Diabetic Mice. Mamm. Genome 2009, 20, 476, doi:10.1007/s00335-009-9217-2.

36. Dorenkott, M.R.; Griffin, L.E.; Goodrich, K.M.; Thompson-Witrick, K.A.; Fundaro, G.; Ye, L.; Stevens, J.R.; Ali, M.; O'Keefe, S.F.; Hulver, M.W.; et al. Oligomeric Cocoa Procyanidins Possess Enhanced Bioactivity Compared to Monomeric and Polymeric Cocoa Procyanidins for Preventing the Development of Obesity, Insulin Resistance, and Impaired Glucose Tolerance during High-Fat Feeding. J. Agric. Food Chem. 2014, 62, 2216-2227, doi:10.1021/jf500333y.

37. Bowser, S.M.; Moore, W.T.; McMillan, R.P.; Dorenkott, M.R.; Goodrich, K.M.; Ye, L.; O'Keefe, S.F.; Hulver, M.W.; Neilson, A.P. High-Molecular-Weight Cocoa Procyanidins Possess Enhanced Insulin-Enhancing and Insulin Mimetic Activities in Human Primary Skeletal Muscle Cells Compared to Smaller Procyanidins. J. Nutr. Biochem. 2017, 39, 48-58, doi:10.1016/j.jnutbio.2016.10.001.

38. Lee, C.; Kim, H.J.; Chang, T.I.; Kang, E.W.; Joo, Y.S.; Kim, H.W.; Park, J.T.; Yoo, T.-H.; Kang, S.-W.; Han, S.H. Synergic Association of Diabetes Mellitus and Chronic Kidney Disease with Muscle Loss and Cachexia: Results of a 16-Year Longitudinal Follow-up of a Community-Based Prospective Cohort Study. Aging 2021, 13, 21941.

39. Mesinovic, J.; Zengin, A.; De Courten, B.; Ebeling, P.R.; Scott, D. Sarcopenia and Type 2 Diabetes Mellitus: A Bidirectional Relationship. Diabetes Metab. Syndr. Obes. Targets Ther. 2019, 12, 1057.

40. Trierweiler, H.; Kisielewicz, G.; Hoffmann Jonasson, T.; Rasmussen Petterle, R.; Aguiar Moreira, C.; Zeghbi Cochenski Borba, V. Sarcopenia: A Chronic Complication of Type 2 Diabetes Mellitus. Diabetol. Metab. Syndr. 2018, 10, 25, doi:10.1186/s13098-018-0326-5.

41. Gu, Y.; Yu, S.; Park, J.Y.; Harvatine, K.; Lambert, J.D. Dietary Cocoa Reduces Metabolic Endotoxemia and Adipose Tissue Inflammation in High-Fat Fed Mice. J. Nutr. Biochem. 2014, 25, 439-445, doi:10.1016/j.jnutbio.2013.12.004.

42. Gu, Y.; Yu, S.; Lambert, J.D. Dietary Cocoa Ameliorates Obesity-Related Inflammation in High Fat-Fed Mice. Eur. J. Nutr. 2014, 53, 149-158, doi:10.1007/s00394-013-0510-1.

43. Goya, L.; Martín, M.Á.; Sarriá, B.; Ramos, S.; Mateos, R.; Bravo, L. Effect of Cocoa and Its Flavonoids on Biomarkers of Inflammation: Studies of Cell Culture, Animals and Humans. Nutrients 2016, 8, 212, doi:10.3390/nu8040212.

44. Griffin, L.E.; Essenmacher, L.; Racine, K.C.; Iglesias-Carres, L.; Tessem, J.S.; Smith, S.M.; Neilson, A.P. DietInduced Obesity in Genetically Diverse Collaborative Cross Mouse Founder Strains Reveals Diverse Phenotype Response and Amelioration by Quercetin Treatment in 129S1/SvImJ, PWK/EiJ, CAST/PhJ, and WSB/EiJ Mice. J. Nutr. Biochem. 2021, 87, 108521, doi:10.1016/j.jnutbio.2020.108521.

45. Bornfeldt, K.E.; Kramer, F.; Batorsky, A.; Choi, J.; Hudkins, K.L.; Tontonoz, P.; Alpers, C.E.; Kanter, J.E. A Novel Type 2 Diabetes Mouse Model of Combined Diabetic Kidney Disease and Atherosclerosis. Am. J. Pathol. 2018, 188, 343-352, doi:10.1016/j.ajpath.2017.10.012.

46. Albrecht, T.; Schilperoort, M.; Zhang, S.; Braun, J.D.; Qiu, J.; Rodriguez, A.; Pastene, D.O.; Krämer, B.K.; Köppel, H.; Baelde, H.; et al. Carnosine Attenuates the Development of Both Type 2 Diabetes and Diabetic Nephropathy in BTBR Ob/Ob Mice. Sci. Rep. 2017, 7, 44492, doi:10.1038/srep44492. 
47. Bitner, B.F.; Ray, J.D.; Kener, K.B.; Herring, J.A.; Tueller, J.A.; Johnson, D.K.; Freitas, C.M.T.; Fausnacht, D.W.; Allen, M.E.; Thomson, A.H. Common Gut Microbial Metabolites of Dietary Flavonoids Exert Potent Protective Activities in $\beta$-Cells and Skeletal Muscle Cells. J. Nutr. Biochem. 2018, 62, 95-107.

48. Fasano, A. Zonulin, Regulation of Tight Junctions, and Autoimmune Diseases. Ann. N. Y. Acad. Sci. 2012, 1258, 25-33, doi:10.1111/j.1749-6632.2012.06538.x.

49. Lau, E.; Carvalho, D.; Pina-Vaz, C.; Barbosa, J.-A.; Freitas, P. Beyond Gut Microbiota: Understanding Obesity and Type 2 Diabetes. Hormones 2015, 14, 358-369.

50. Nagpal, R.; Newman, T.M.; Wang, S.; Jain, S.; Lovato, J.F.; Yadav, H. Obesity-Linked Gut Microbiome Dysbiosis Associated with Derangements in Gut Permeability and Intestinal Cellular Homeostasis Independent of Diet. J. Diabetes Res. 2018, 2018.

51. Jayashree, B.; Bibin, Y.S.; Prabhu, D.; Shanthirani, C.S.; Gokulakrishnan, K.; Lakshmi, B.S.; Mohan, V.; Balasubramanyam, M. Increased Circulatory Levels of Lipopolysaccharide (LPS) and Zonulin Signify Novel Biomarkers of Proinflammation in Patients with Type 2 Diabetes. Mol. Cell. Biochem. 2014, 388, $203-210$.

52. Gil-Cardoso, K.; Ginés, I.; Pinent, M.; Ardévol, A.; Blay, M.; Terra, X. Effects of Flavonoids on Intestinal Inflammation, Barrier Integrity and Changes in Gut Microbiota during Diet-Induced Obesity. Nutr. Res. Rev. 2016, 29, 234-248.

53. Sanz-Lamora, H.; Marrero, P.F.; Haro, D.; Relat, J. A Mixture of Pure, Isolated Polyphenols Worsens the Insulin Resistance and Induces Kidney and Liver Fibrosis Markers in Diet-Induced Obese Mice. Antioxidants 2022, 11, 120, doi:10.3390/antiox11010120.

54. Sato, A.Y.; Pellegrini, G.G.; Cregor, M.; McAndrews, K.; Choi, R.B.; Maiz, M.; Johnson, O.; McCabe, L.D.; McCabe, G.P.; Ferruzzi, M.G.; et al. Skeletal Protection and Promotion of Microbiome Diversity by Dietary Boosting of the Endogenous Antioxidant Response. J. Bone Miner. Res. 2021, 36, 768-778, doi:10.1002/jbmr.4231.

55. Preguiça, I.; Alves, A.; Nunes, S.; Fernandes, R.; Gomes, P.; Viana, S.D.; Reis, F. Diet-Induced Rodent Models of Obesity-Related Metabolic Disorders-A Guide to a Translational Perspective. Obes. Rev. 2020, 21, e13081, doi:10.1111/obr.13081.

56. Kanasaki, K.; Koya, D. Biology of Obesity: Lessons from Animal Models of Obesity. J. Biomed. Biotechnol. 2011, 2011, e197636, doi:10.1155/2011/197636.

57. Medina-Contreras, J.; Villalobos-Molina, R.; Zarain-Herzberg, A.; Balderas-Villalobos, J. Ovariectomized Rodents as a Menopausal Metabolic Syndrome Model. A Minireview. Mol. Cell. Biochem. 2020, 475, 261-276, doi:10.1007/s11010-020-03879-4.

58. Davidge, S.T.; Zhang, Y.; Stewart, K.G. A Comparison of Ovariectomy Models for Estrogen Studies. Am. J. Physiol.Regul. Integr. Comp. Physiol. 2001, 280, R904-R907, doi:10.1152/ajpregu.2001.280.3.R904.

59. Diaz Brinton, R. Minireview: Translational Animal Models of Human Menopause: Challenges and Emerging Opportunities. Endocrinology 2012, 153, 3571-3578, doi:10.1210/en.2012-1340.

60. Iglesias-Carres, L.; Neilson, A.P. Utilizing Preclinical Models of Genetic Diversity to Improve Translation of Phytochemical Activities from Rodents to Humans and Inform Personalized Nutrition. Food Funct. 2021, doi:10.1039/D1FO02782D.

61. Racine, K.C.; Wiersema, B.D.; Griffin, L.E.; Essenmacher, L.A.; Lee, A.H.; Hopfer, H.; Link to external site, this link will open in a new window; Lambert, J.D.; Link to external site, this link will open in a new window; Stewart, A.C.; et al. Flavanol Polymerization Is a Superior Predictor of $\alpha$-Glucosidase Inhibitory Activity Compared to Flavanol or Total Polyphenol Concentrations in Cocoas Prepared by Variations in Controlled Fermentation and Roasting of the Same Raw Cocoa Beans. Antioxid. Basel Switz. 2019, 8, doi:http://dx.doi.org.prox.lib.ncsu.edu/10.3390/antiox8120635. 
62. Racine, K.C.; Lee, A.H.; Wiersema, B.D.; Huang, H.; Lambert, J.D.; Stewart, A.C.; Neilson, A.P. Development and Characterization of a Pilot-Scale Model Cocoa Fermentation System Suitable for Studying the Impact of Fermentation on Putative Bioactive Compounds and Bioactivity of Cocoa. Foods 2019, 8, 102, doi:10.3390/foods8030102.

63. Goodrich, K.M.; Neilson, A.P. Simultaneous UPLC-MS/MS Analysis of Native Catechins and Procyanidins and Their Microbial Metabolites in Intestinal Contents and Tissues of Male Wistar Furth Inbred Rats. J. Chromatogr. B 2014, 958, 63-74, doi:10.1016/j.jchromb.2014.03.011.

64. Reagan-Shaw, S.; Nihal, M.; Ahmad, N. Dose Translation from Animal to Human Studies Revisited. FASEB J. 2008, 22, 659-661, doi:10.1096/fj.07-9574LSF. 